\title{
What have we learned from helioseismology, what have we really learned, and what do we aspire to learn?**
}

\author{
Douglas Gough
}

(C) Springer $\bullet \bullet \bullet$

\begin{abstract}
Helioseismology has been widely acclaimed as having been a great success: it appears to have answered nearly all the questions that we originally asked, some with unexpectedly high precision. We have learned how the sound speed and matter density vary throughout almost all of the solar interior which not so very long ago was generally considered to be impossible - we have learned how the Sun rotates, and we have a beautiful picture, on a coffee cup, of the thermal stratification of a sunspot, and also an indication of the material flow around it. We have tried, with some success at times, to apply our findings to issues of broader relevance: the test of the General Theory of Relativity via planetary orbit precession (now almost forgotten because the issue has convincingly been closed, albeit no doubt temporarily), the solar neutrino problem, the manner of the transport of energy from the centre to the surface of the Sun, the mechanisms of angular-momentum redistribution, and the workings of the solar dynamo. The first two were of general interest to the broad scientific community beyond astronomy, and were, quite rightly, principally responsible for our acclaimed success; the others are still in a state of flux.
\end{abstract}

Keywords: Helioseismology, Heliophysics; Solar neutrinos; General Relativity; Solar opacity; Equation of state

\footnotetext{
*Invited Article

Institute of Astronomy, Madingley Road, Cambridge, CB3

0HA; Department of Applied Mathematics and Theoretical

Physics, Centre for Mathematical Sciences, Wilberforce

Road, Cambridge, CB3 0WA, UK

(e-mail: douglas@ast.cam.ac.uk);

Physics Department, Stanford University, CA 94305, USA
} 


\section{Prelude}

In the early heady days of helioseismology, the new techniques of inference, coupled with pertinent observations that had been stimulated by them, went hand-in-hand with consequent scientific discovery, and helioseismology was not unnaturally perceived to be almost a branch of science. Now, in its relative maturity, it is, or at least it should be, relegated to what it really is: a very valuable technique for drawing scientific inference. The basic physics of seismic oscillations is not new, is fundamentally quite simple, and is well understood; and the principles of inference from the observations should be straightforward to comprehend, even though the technicalities of putting them into practice may for some seem to be rather complicated. Therefore the robust raw conclusions are genuinely secure, more so than the broader issues to which they are intended to be applied. However, it is incumbent upon us to make the distinction between the inferences that really have been drawn reliably and the further wider inferences that might subsequently be, or have been, drawn, often with the aid of supplementary, possibly less secure, maybe non-seismic, information, and even, maybe, (sometimes unstated) surmise. Only if such distinction is made clear can the contributions of our subject to science be reaped to the full. Unfortunately, that aspiration has not always been achieved in the past, and misinformation has sometimes sullied the waters.

It takes only a brief scrutiny of the equations describing the structure and dynamical evolution of the Sun (it is not quite so brief to derive them) and the equations governing the low-amplitude seismic modes of oscillation to appreciate what broadly can, at least in principle, be reliably inferred. Anything further must depend on other criteria, such as general physical argument beyond seismology, traditional astronomical observation, or even prejudice. It is obligatory to be explicit about how such additional constraints are applied. The subject has advanced to a new level of sophistication; we are now trying to probe seismically (and otherwise) almost inaccessible aspects of the physics of the Sun, and the techniques for unravelling them are becoming more and more intricate, beyond the point at which most scientists wish to tread. There must necessarily be an increased trust in our findings, and it is our responsibility not to betray it. Many of the broader scientific community want to use our results in their research; for that they need to know not only the limitations of our inferences, and the caveats upon which they are based, but also which aspects of what we seismologists tell them can really be trusted.

Much of the emphasis of Solar Dynamics Observatory seismology concerns the workings of the convection zone. We want to know what controls the solar cycle, how magnetic field is amplified, modulated, and then suppressed, how sunspots are formed and destroyed - and what determines their lifespan. We want to know the geometry of at least the larger scales of convective motion, and how, beneath the seen superficial layers of the Sun, the processes that control the total radiative output are modulated. At least some of us want to understand 
how all these matters influence our procedures for inferring the gross properties of the Sun, and how they impinge on our broader ideas of the evolution of the Sun in particular, and of stars in general. Addressing such delicate issues with confidence may now seem an almost impossible task to us older scientists who have lived through the years of stumbling through the darkness, having finally emerged to bathe in the secure light illuminating the minute arena of knowledge that we have been instrumental in uncovering. It is now up to the younger community to proceed likewise: to grasp at the edge of our perception with initially insecure ideas, fully appreciating the uncertainty, of course; then moulding and strengthening them into a new body of secure scientific knowledge.

\section{Introduction}

Once the potential of solar oscillations to map the interior of the Sun was recognized (Christensen-Dalsgaard and Gough, 1976) there were two obvious serious issues that immediately appeared accessible to resolution: the spherically symmetric component of the hydrostatic stratification and the internal angular velocity. The first of these was needed for investigating what has been called the solar neutrino problem; the second concerned the centrifugally induced oblateness of the Sun's gravitational equipotentials, and how that impinged upon an important test of theories of gravity, General Relativity in particular, via the precession of planetary orbits. These were the two most widely discussed issues in heliophysics at the time.

The stratification was originally addressed with the help of theoretical models. Deubner (1975) had published the first well resolved $k-\omega$ spectrum. and Ando and Osaki (1975) had shown that theoretical eigenfrequencies of p modes trapped in the outer layers of a solar model envelope were in quite good, but not perfect, agreement with Deubner's observations. What was required for bringing the theory more closely into line with observation was first estimated from the properties of the eigenfrequencies of a simple polytropic representation of the outer layers of the convection zone as a means of calibrating solar models (Gough, 1977); the conclusion was that the convection zone must be about $200 \mathrm{Mm}$ deep, some $50 \mathrm{Mm}$ deeper than the favoured value of the time. Basically, that conclusion was drawn from relating theoretical eigenfrequencies to the jump in the adiabatic "constant" $p / \rho^{\gamma_{1}}$, which is closely related to specific entropy, across the thin superadiabatic convective boundary layer. It was subsequently supported by more realistic, numerical, computations by Ulrich and Rhodes (1977). The principal implication of that result was that, according to complete solar models, a deeper convection zone implied greater helium and heavy-element abundances, a hotter, more centrally condensed, core, and a higher neutrino flux, thereby exacerbating the solar neutrino problem (e.g. Abraham and Iben, 1971; Bahcall and Ulrich, 1971). Indeed, one is tempted to speculate that the modellers in the past had adjusted the defining parameters of their models to minimize 
the theoretical neutrino flux, and that that had prejudiced Ando and Osaki's calculations, although without repeating the calculations oneself (and maybe even if one did) one cannot be sure.

There was a great deal of healthy mistrust in the models at that time. The concern was that a seismological measurement - I should really say estimate of merely the upper boundary layer of the convection zone, extending only a minute fraction of the solar radius beneath the photosphere, could hardly be a robust indicator of conditions inside the energy-generating core. Therefore, as soon as low-degree data became available (Claverie et al. . 1980: Grec, Fossat, and Pomerantz, 1980; Fossat. Grec, and Pomerantz, 1981) it became possible to use seismic indicators of more global properties; first the so-called large frequency separation (Christensen-Dalsgaard, Gough, and Morgan, 1979a. b; ChristensenDalsgaard and Gough, 1980), which measures the sound travel time from the centre of the Sun to the seismic surface1 (e.g. Vandakurov, 1967; Tassoul, 1980; Gough, 1986), and later the small frequency separation (Gough, 1983c), which is a direct indicator of conditions in the core (e.g. Gough, 1983a, 1986). Unfortunately it was not possible to make all of the theoretical frequencies agree with the data, as was clear from an indiscriminant attempt to fit only the low-degree frequencies with whole-disc observations (Christensen-Dalsgaard and Gough, 1981): two solar models seemed to be favoured, one with a low initial helium abundance: $Y_{0}=0.18$ (coupled with a correspondingly low heavy-element abundance $Z=0.003$ and a low neutrino flux, although not low enough to reproduce the neutrino detection rate), the other large: $Y_{0}=0.27$ and $Z_{0}=0.026$. The high- $Y$ model fitted rather better, and one was tempted to prefer it, especially because its high-degree mode frequencies were closer to Deubner's observations. Moreover, the low- $Y$ model had a helium abundance below what was thought to have been produced in the Big Bang ( $c f$. footnote 8), which would call for some contrived explaining.

${ }^{1}$ I consider the seismic surface $r=R$ of the Sun (assumed here to be spherically symmetrical) to be the radius at which $c^{2}$, regarded as a function of $r$, or $c$, regarded as a function of acoustic radius $\tau(r)=\int c^{-1} \mathrm{~d} r$ - both of which are close to being linear functions in the outer adiabatically stratified lavers of the convection zone (Balmforth and Gough, 1990; Lopes and Gough, 2001) - extrapolate to zero. In the Sun, according to Model S of Christensen-Dalsgaard et al. (1996), it lies about $1000 \mathrm{~km}$ above the photosphere, the precise value depending on exactly how the extrapolation is carried out. There is nothing special about the structure of the actual atmosphere in its vicinity, which is well inside the outer evanescent zone of most of the seismic modes and therefore has little significant influence on the dynamics. Instead, it acts simply as a (virtual) singularity in the acoustic wave equation, providing a convenient parametrization of conditions (well below the photosphere) in the vicinity of the upper turning points of the modes. Put another way, it provides a convenient fiducial location with respect to which the acoustic phase in the propagating zone beneath is related. Unlike the photosphere, which has no acoustic significance, it shares a relation with the deeper solar interior that is robust, and is insensitive to the non-seismic, thermal and radiative, properties of the outer convective boundary layer, whose structure changes with the solar cycle Antia and Basu, 2004; Dziembowski and Goode, 2004, 2005: Lefebvre and Kosovichev, 2005; Lefebvre, Kosovichev, and Rozelot, 2007). In contrast to other, non-seismic, radii (cf. (Bahcall and Ulrich, [1988), it provides a stable outer limit to the effective total acoustic-radius integral $\tau(R)$, which determines the large frequency separation; in Model S it is some 200 seconds or so greater than the actual acoustic radius of the photosphere. 
The two models required different identifications of the orders $[n]$ of the modes; unfortunately the orders were so high, and the fit so poor, that reliable extrapolation to $n=1$ was not possible. It was not until Duvall and Harvey (1983) observed the frequencies of modes of intermediate degree that a secure connection between modes of low and high degree could be made Gough, 1983b). The orders of the latter are determinable because the frequencies of high-degree $\mathrm{f}$ modes, with which is associated $n=0$, are essentially independent of the structure of the Sun (Gough, 1982a). The high- $Y$ alternative was thereby confirmed.

Thus we had learned that the solar neutrino problem was almost certainly not resolvable by adjusting solar models, and must be a matter for nuclear or particle physics.

Had we really learned that? Perhaps not yet. At the time there were still very serious doubts about the solar models, for they depended on many unproven assumptions, some of which are listed in Table 1 . There had already been, and there were yet to be, many models computed in which some of these assumptions were relaxed in the hope of yielding lower neutrino fluxes (although none provided a satisfactory reconciliation of theory with observation). At the very least, a secure representation of the stratification throughout the Sun was surely required. That was soon to be provided from inversion analyses of frequency data from Duvall and Harvey (Christensen-Dalsgaard et al.. 1985: Christensen-Dalsgaard, Gough, and Thompson, 1989; (Dziembowski, Pamyatnykh, and Sienkiewicz, 1990), from which it was possible to infer the (spherically averaged) sound speed $[c(r)]$ throughout almost all of the Sun. Details of the core were not yet within reach (and even today there is considerable uncertainty), but elsewhere the sound speed was essentially the same as that of Christensen-Dalsgaard and Gough's (1981) high- $Y$ model. It was also possible to see the base of the convection zone. I recall one Friday morning (the second Friday of January, 1984, a day on which I was due to deliver a lecture on my findings to the Royal Astronomical Society); it was about 4:30 in the morning - one could compute seriously only at night in those days - when I obtained my first plot of $c^{2}(r)$ that extended beneath the convection zone; I plotted $c^{2}$ and not $c$ because it is related closely to temperature $T$ - the equation of state in the solar interior is reasonably well approximated by the perfect-gas law, for which $c^{2} \propto T / \mu$, where $\mu$ is the "mean molecular mass". A more modern (and rather better) version of what I obtained is illustrated in Figure 田. The location of the base of the convection zone is

${ }^{2}$ Included for comparison is one of the models with low $Y$ that was used in the original calibration with low-degree modes by Christensen-Dalsgaard and Gough (1981). The qualitative differences between the models can be appreciated by realizing first that the radiative envelopes are roughly polytropic (with index 3.5), and that it is adequate to approximate the equation of state by the perfect-gas law. Then it is clear that the magnitude of $T(r)$ (and $\rho$ ) must be greater in the higher- $Y$ model, because the total energy generation rate, which is an increasing function of $X, \rho$, and $T$, is the same for the two models. Polytropic scaling (e.g. Gough, 1990) indicates that $Z$ is a steeply decreasing function of $X$, so $Z$ is much greater in the higher- $Y$ model. In addition, the polytropic radius scale is greater for the higher- $Y$ model, as is evident 

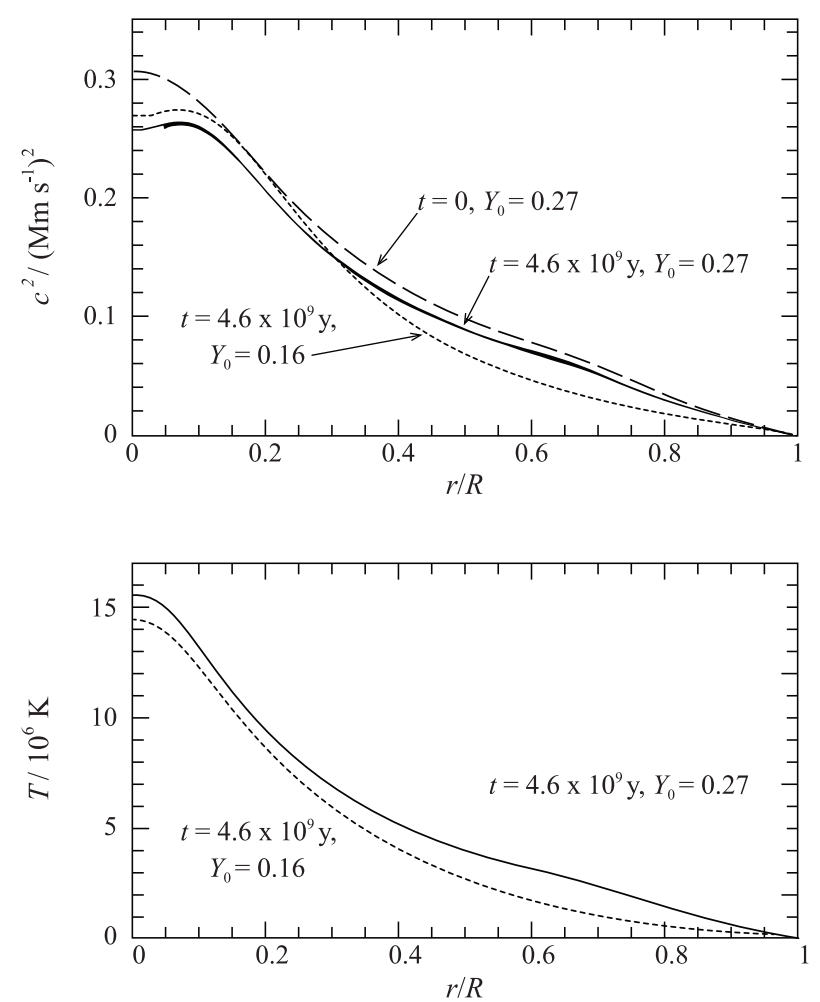

Figure 1. Upper panel: The continuous curve extending over the entire range of $r / R$ is the square of the sound speed $\left[c^{2}\right]$ in the standard solar model $\mathrm{S}$ of Christensen-Dalsgaard et al. (1996), which was computed with initial hydrogen and heavy-element abundances $X_{0}=0.7091, Z_{0}=0.0196$. The square of the sound speed in the Sun is also plotted (where $r / R>0.1$ ) as a continuous curve; the difference between the two can be barely discerned by the variation in the thickness of the apparently single curve (but see Figures 3 and 44). The dip in $c^{2}$ at the centre of the Sun is a result of the augmentation of $\mu$ by nuclear transmutation, which increases with time on the main sequence (and which can therefore be used as a diagnostic of main-sequence age). The dashed curve is $c^{2}$ on the zero-age main sequence, and, except near the surface where abundant elements undergo ionization, is (locally) proportional to temperature. The dotted curve is $c^{2}$ in a model with an initial heavy-element abundance $Z=0.001$, but continuously contaminated at the surface at such a constant rate as to have a current heavy-element abundance $Z_{\mathrm{s}}=0.02$ in the convection zone today (Christensen-Dalsgaard, Gough, and Morgan, 1979a). Lower panel: Temperature $[T]$ through the two present-day solar models.

evident as a near discontinuity of the second derivative, as one can see more

from the Figure 1 by imagining an extrapolation of the functional form of $T(r)$ outwards from the radiative zone. Consequently the convection zone, which steepens the gradient, has more truncating from the radiative structure to perform in order to maintain the observed photospheric radius, and is therefore deeper. An additional scaling in magnitude is required to convert $T$ to $c^{2} \propto T / \mu$, raising the dotted curve (corresponding to the model with the lower $Y$ ) relative to the continuous curve. It also depresses both curves near the centre of the star, in the energy-generating core where $\mu$ has been augmented by nuclear transmutation, providing a diagnostic of main-sequence age. 
easily by holding the page almost in one's line of sight and looking along the curve. I confirmed the location by having a research student, the only person left in the computer room at that hour, repeat the exercise. I went home, slept a couple of hours, and then took a train to London. When I showed the plot at the RAS (Gough, 1984a) and pointed out the discontinuity, the audience was incredulous, even though I stood almost in the plane of the screen to reassure them. I then recounted the confirmation by the research student, and there was a sudden release of tension in the audience when I told them that the student was a cosmologist, for then they appreciated that such a student must undoubtedly have provided an unbiased opinion on such a matter.

The result was compared with theoretical solar models (Christensen-Dalsgaard et al., 1985), and revealed a characteristic discrepancy (much larger than that evident in the more modern comparisons, such as those illustrated in Figures 3 and (4) immediately beneath the base of the convection zone. It was suggested that the discrepancy could have been caused by an error in the opacity (values of opacity in those days were available in tables provided by the Los Alamos National Laboratory in the USA). Such suggestions, in a rather broad sense, had been made in the past, for example by Simon (1982) in response to a failure to reproduce simultaneously observations relating to stellar evolution and observations of pulsational characteristics of classical variable stars. But here one was able to state quite precisely the thermodynamical conditions under which the opacity was in error, and the approximate magnitude (and sign) of the error. After some persuasion, Carlos Iglesias and Forrest Rogers at Livermore computed by moonlight the opacity for a few judiciously chosen values of the state variables $\rho$ and $T$ (and a plausibly appropriate chemical composition) using an independently written computer programme; they not only confirmed the helioseismic inference but also showed that the discrepancy was even greater at lower temperatures - of no concern for the Sun because it is well inside the adiabatically stratified region of the convection zone where radiative energy transfer is negligible, but of great significance in reconciling with observation the theory of some classes of intrinsically variable stars such as $\beta$ Cephei and slowly pulsating B stars (Cox et al.. 1992: Kiriakidis. El Eid, and Glatzel., 1992: Moskalik and Dziembowski, 1992; Dziembowski and Pamvatnvkh. 1993: Dziembowski, Moskalik, and Pamyatnvkh. 1993. 1994) and double-mode Cepheids (Moskalik, Buchler, and Marom, 1992). Largely as a result of efforts by Werner Däppen, scientists who were involved with the Los Alamos opacity computations were brought into the same room as the Livermore scientists, and errors in the Los Alamos calculations were identified. This led to the Lawrence Livermore National Laboratory providing the funds for more extensive opacity computations for astrophysical use (Iglesias. Rogers, and Wilson, 1990; Iglesias and Rogers, 1991, 1996; Rogers and Navfonov, 2002). Thus we see the first example of helioseismology contributing directly to microscopic physics (if one considers the neutrino issue to be indirect). There have been further contributions, but I postpone discussion of those until later.

I emphasize that an important consequence of the sound-speed inversion was that it convincingly ruled out the low- $Y$ models, reaffirming that the cause of 
the neutrino deficit was not in solar modelling. That conclusion was not fully appreciated by many of the solar modellers of the time, for they had not yet understood the power of helioseismological analysis.

I turn now to the angular velocity $[\Omega]$, which is measured by the odd (with respect to azimuthal order $m$ ) component of degeneracy splitting caused by advection and Coriolis acceleration. Strictly speaking, one needs the sound-speed inversion first, to establish the hydrostatic stratification with respect to which the splitting kernels are computed. However, the angular velocity was actually first obtained by using a theoretical solar model before the full sound-speed inversion had been carried out (Duvall et al., 1984), partly, perhaps, because it was easier to perform with the data in hand, yet also with the knowledge that the error from which that procedure suffered was much less than that resulting from the errors in the measurements of the degeneracy splitting. The result was a big surprise:

There had been many prior discussions of how much faster than the photosphere the solar core must be rotating (due to spin-down), the most widely publicized being the discussions resulting from the measurements of the apparent oblateness of the solar surface by Dicke and Goldenberg (1967, 1974). The oblateness measurements had been made in the hope of confirming Dicke's theory of gravitation (Brans and Dicke, 1961) 3: put naively, in the Brans-Dicke

\footnotetext{
${ }^{3}$ The gravitational attraction associated with the energy density $-G M / r$ of the gravitational field surrounding the Sun, absent in Newton's theory, causes the total gravitational attraction to increase: very roughly speaking, as a result of energy conservation the apparent gravitational mass of a planet at distance $r$ from the Sun, in a flat representation of space, is augmented by approximately $G M / c^{2} r$ per unit mass above what it would have appeared to have been at infinity; $M$ is the mass of the Sun, and here $c$ is the speed of light. Similarly, the energy, hence the frequency, of a photon is multiplied by a factor $\Gamma=1+G M / c^{2} r-$ that causes the familiar gravitational redshift. Consequently, the orbit equation is modified simply by multiplying the Newtonian gravitational force on the planet by $\Gamma^{3}$. After linearization and rewriting $M$ in terms of the orbital specific angular momentum $h=\sqrt{(} G M r)$, valid for nearly circular orbits, the effective attractive force becomes $-\left(1+3 h^{2} / c^{2} r^{2}\right) G M / r^{2}$; it increases with increasing proximity more rapidly than Newton's inverse square. It is easy to see also that the gravitational field in the equatorial plane of a rotating (oblate) axisymmetric self-gravitating body (like the Sun) also increases with decreasing distance faster than the field around a corresponding spherically symmetrical body: the act of flattening a spherical body takes equal amounts of material from the poles towards the near and the far sides of the equator, the increase in gravitational attraction by closer nearside matter exceeding the lesser decrease by farside matter. Therefore the net gravitational attraction is increased, by an amount which inceases as $r$ decreases and the shape of the Sun becomes more apparent. The force is given approximately by $-\left(1+\frac{3}{2} J_{2} R^{2} / r^{2}\right) G M / r^{2} ; J_{2}$ is the quadrupole moment. In both cases, therefore, a planet is drawn towards the Sun increasingly strongly with increasing proximity than it would have been in an inverse-square field. Conserving its angular momentum, it is thereby caused to rotate through a greater angle near perihelion because its orbital angular velocity is augmented, distorting an otherwise approximately elliptical bound Newtonian orbit in such a manner as to appear to make it simply precess in the same direction as the angular velocity of the planet. (Near aphelion the oppositely directed contribution to the precession is lesser, therefore too small to annul the contribution from near perihelion.) Use of planetary (or spacecraft) orbital precession rates to calibrate the relativistically induced deviation from the inverse-square gravitational field surrounding the Sun therefore requires one to know the contribution from the distortion from spherical symmetry of the mass distribution in the Sun,
} 
theory Newton's gravitational constant $[G]$ was regarded as a field satisfying a wave equation that couples to the matter, thereby relaxing the gravitational force field from the relative rigidity that is imposed when $G$ is held constant, and hence reducing the rate of precession of planetary orbits from the value predicted by General Relativity; the fact that General Relativity predicts essentially the correct precession with a spherical Sun was regarded by Dicke as a fortuitous coincidence, and that in reality the shortfall predicted by his theory (which is not an absolute prediction, but depends on an unknown coupling constant) is made up by the oblateness of the Sun's gravitational field produced by a rapidly rotating interior. The oblateness measurements would calibrate the coupling constant. A debate ensued concerning two matters: the relation between the oblateness in surface brightness and the oblateness of the gravitational equipotentials (e.g. Dicke, 1970), and the fluid dynamics of the solar interior concerning angular-momentum transfer from centre to surface during spin-down (e.g. Howard. Moore. and Spiegel, 1967; Dicke, 1967; Bretherton and Spiegel, 1968). I shall enlarge on neither here, despite the intrinsic interest of each, because even the early seismological analysis (Duvall et al., 1984) revealed that the Sun is unexpectedly rotating almost uniformly throughout its interior, except possibly in what might be its almost inaccessible core (Figure 21). Subsequent observations of low-degree modes revealed that the core rotates significantly no more rapidly than the envelope (Elsworth et al., 1995; Chaplin et al., 1999). From knowledge of $\Omega$ and the density and pressure stratification, the multipole moments $J_{2 k}$ of the gravitational equipotentials can easily be computed (e.g. Gough, 1981; Pijpers, 1998). I maintain that this is the most accurate way to determine $J_{2 k}$, notwithstanding some claims in the literature to the contrary. The reason is partly that other methods involve relating surface brightness asphericity with gravity, which is not well understood, and partly because those measures rely on only the small quadratic centrifugal force rather than the more robust, and unambiguous, seismic effects of advection and, to a much lesser degree, Coriolis acceleration, both of which are predominantly linear in $\Omega$ and are therefore much larger than the centrifugal term; and, moreover, they do not depend on relating brightness to gravity. Moreover, the rotational distortion of the isobaric surfaces in the vicinity of the photosphere is dominated by the direct effect of the local centrifugal force, and exceeds the gravitational distortion by a factor of more than 20; determining the latter from the apparent shape of the solar disc therefore requires a subtraction of two measurements that differ by less than a mere 5 per cent. Even the most precise limb-shape measurements (e.g. Fivian et al., 2008; Kuhn et al., 2012) are far from that goal (Gough, 2012b).

The outcome is consistent with General Relativity; indeed, the miniscule contribution that $J_{2}$ does make to the precession of the orbit of Mercury (e.g. Gough, 1982b; Duvall et al., 1984; Brown et al., 1989; Antia. Chitre. and Gough, 2008) brought General Relativity into somewhat closer agreement with observation than assuming the Sun to be spherically symmetrical. The influence of higher

such as is produced by centrifugal acceleration due to rotation. The precession rate is most easily calculated by perturbation theory (e.g. Ramsev, 1937). 


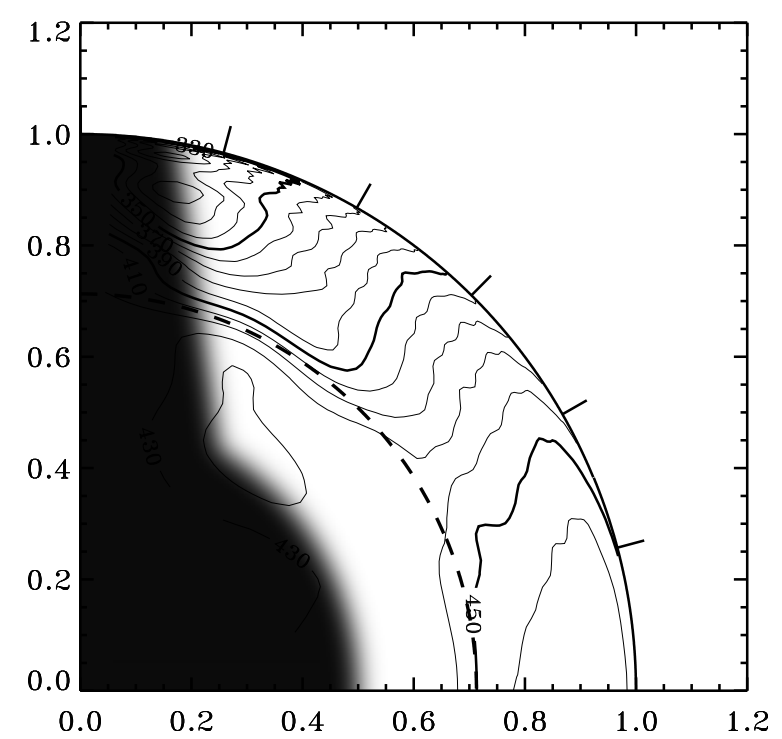

Figure 2. Optimally localized averages of the North-South symmetric component of the angular velocity $[\Omega]$ of the Sun, depicted as a contour plot in a quadrant. Some contours are labelled [nHz], and, for clarity, every fifth contour is drawn bold; the contour separation is $10 \mathrm{nHz}$. The outer quarter circle denotes the surface of the Sun, the dashed quarter circle indicates the base of convection zone, and the tick marks at the Sun's surface are drawn at latitudes $15,30,45,60$, and $75^{\circ}$. The Equator is the horizontal (relative to the page) axis and the Pole the vertical, each labelled with values of $x=r / R$. The shaded area indicates the region in the Sun where no reliable inference could be made from the data available (from Schou et al., 1998). There has been little significant improvement of this inference, save for a demonstration that the core is not rotating rapidly (Elsworth et al., 1995; Chaplin et al., 1999); however, there have been studies of temporal variation associated with the solar cycle (e.g. Vorontsov et al., 2002; Basu and Antia, 2003; : Howe et al., 2006a b;: Antia, Chitre, and Gough, 2008).

moments is utterly negligible. The improvement is marginal, but with future, more delicate, orbital analyses the helioseismological determination of $J_{2}$ will evidently take on a crucial role. The almost uniform rotation of the radiative interior of the Sun begs the question of how that can be, particularly because it interfaces with a differentially rotating convection zone. I shall return to that matter later.

\section{Understanding Seismic Variables}

It is extremely important to understand what is actually inferred from seismology, for only then can one draw reliable conclusions concerning the Sun. Seismic modes are essentially adiabatic. They result from forces - pressure and 
gravity predominantly - acting on matter with inertia. The dynamics therefore concerns only pressure $[p]$ and matter density $[\rho]$ which are related via gravity through hydrostatic equilibrium, and the relation between perturbations to them under adiabatic change, which is characterized by the first adiabatic exponent $\left[\gamma_{1}=(\partial \ln p / \partial \ln \rho)_{s}\right.$, the partial thermodynamic derivative being taken at constant specific entropy $[s]]$. The restoring force of acoustic modes (p modes) is principally pressure, that of gravity modes (g modes, including the fundamental $\mathrm{f}$ modes) is buoyancy. So the modes can provide information directly about only $p, \rho$, and $\gamma_{1}$ (and, of course, any function of them). These are the basic seismic variables. I should acknowledge that any magnetic field [B] that is present also contributes to the dynamics, and therefore is also a seismic variable. Unfortunately, its effect on the frequencies of the modes appears to be indistinguishable from that of an appropriate variation in sound speed $[c]-$ also a seismic variable because $c^{2}=\gamma_{1} p / \rho$ - which makes it difficult to unravel the two (Zweibel and Gough, 1995). In principle one might be able to do so from the eigenfunctions, which are configured differently by $c$ and $\mathbf{B}$, although supplementation by non-seismic arguments is likely to be more productive.

Leaving $\mathbf{B}$ aside for the time being, it should be appreciated that the relation between $p$ and $\rho$ through hydrostatics does not depend directly on $\gamma_{1}$, so $p$ or $\rho$ (or any function of only them) and $\gamma_{1}$ are structurally independent: it follows that although the relation between them, which physically is given by the equation of state, can be determined by seismology along the thermodynamic $p$ $\rho$ path through the Sun, there is no remaining redundancy, so the veracity of the equation of state itself cannot therefore be probed by seismology alone; in order to investigate the equation of state, supplementary, non-seismic, information is required.

There are two approaches that one can take for drawing seismic inference. One is to adopt a parametrized model of the Sun, or some aspect of it, and from it calculate whatever seismic properties one wishes to compare with observation. The comparison calibrates the controlling parameters. That was the procedure that I described in my introduction for first estimating the depth of the convection zone, and consequently the helium abundance. The other approach is to ignore explicit models (almost) entirely, and combine the data in such a manner as to isolate certain properties of the seismic structure. Typically that involves adopting, at least at first, a reference model of the Sun, which it is hoped is sufficiently close to the Sun for linearization in the small differences from it to be more-or-less valid; that simplifies the analysis enormously. Subsequent iteration can usually remove the dependence on the reference model. Nonlinear asymptotic methods have also been used successfully to yield approximate inferences without recourse to a reference model at all. The most common procedures that have been used to date are aimed at obtaining easily interpretable representations of the basic seismic variables. They are commonly called inversions.

When linearization about a reference model is carried out, the frequency differences between the Sun and the model can each be expressed as a sum 
of spatial averages of independent seismic variables. A prudent procedure is then to seek suitable linear combinations of those averages which more easily inform one of whatever question one has chosen to pose. If it is the value of a particular seismic variable that one wants to investigate, then the most easily interpretable data combinations are those that represent averages of that variable with weighting functions (called kernels in this context) that are highly localized with hardly any sidelobes, and which at the same time are (almost) independent of any other variable, for then the result can be thought of as a blurred view of the variable of interest. Consequently one tries to tailor maximally localized kernels, although the attempted maximization must be moderated by a requirement that the interference from extraneous seismic variables be kept low and that the influence of data errors not be excessive. On the whole, increasing localization requires data combinations with coefficients of greater and greater magnitude, which increases the influence of random data errors. The procedure to construct those kernels is now called optimally localized averaging (OLA). Just how low one demands the interference from other variables to be, and how much the influence of data errors one considers to be acceptable, is a matter of personal choice, which probably explains why the errors in the averages displayed in Figures 3 and 4 differ. This kind of tailoring was the first to be advocated for helioseismology (Gough, 1978), in contrast to the almost universal opinion of geoseismologists of the time who objected to the procedure on the ground that one might be tempted simply to draw a curve through the averages and mistake that curve for the actual variable, rather than the average that it actually is. We helioseismolgists usually do not make that mistake. We explicitly draw a sequence of crosses, as in Figure 3 the horizontal components representing the averaging widths, the vertical bars representing the propagated standard errors in the data. Some geoseismologists are now changing their tune, and are following suit.

An alternative procedure, common in geoseismology, is to seek a putative "solution" that reproduces the data. Typically, one first expresses the seismic variables as linear combinations of a prescribed set of basis functions, and then one chooses the coefficients in each combination to match the data the most closely, again moderated (regularized) to avoid excessive error magnification. The reason geoseismologists used to prefer that approach is that it might yield a curve that actually fits the data within the estimated errors, which OLA might not (and is not explicitly designed to). If it is a linearized perturbation to a reference model that is being fitted to the data, the outcome can also be expressed as averages with localized components. However, the averaging kernels often have severe sidelobes too, usually near the surface of the Sun, which renders interpretation more difficult. Moreover, the "solution", if it exists, is just one of infinitely many that satisfy the constraints imposed by the data. Usually, error-correlation information about the raw data is unavailable, and the data are usually fit by (regularized) least squares (RLS), regularization being accomplished typically by imposing some criterion of smoothness on the ground that the data provide only finite spatial resolution. 

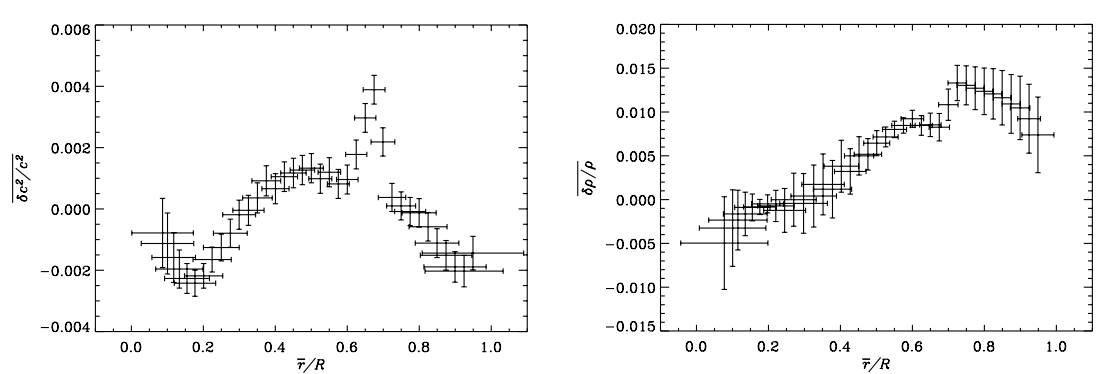

Figure 3. Optimally localized averages of the relative differences of the squared sound speed $\left[c^{2}\right]$ and the density $[\rho]$ in the Sun from those in Model S of Christensen-Dalsgaard et al. (1996), computed by M. Takata (Takata and Gough, 2001) from MDI 360-day data and plotted against the centres $[\bar{x}=\bar{r} / R]$ of the averaging kernels $[A(x ; \bar{x})]$ (which here resemble Gaussian functions), defined by $\bar{x}=\int x A^{2} \mathrm{~d} x / \int A^{2} \mathrm{~d} x$. The length of each horizontal bar is twice the spread $s$ of the corresponding averaging kernel, defined as $s=12 \int(x-\bar{x})^{2} A^{2} \mathrm{~d} x$ - an averaging kernel $A$ that is well represented by a Gaussian function of variance $\Delta^{2}$ has spread approximately $1.7 \Delta \approx 0.72 \mathrm{FWHM}$; were it to be a top-hat function, its spread would be the full width, which is why $s$ has been so defined. The vertical bars extend to \pm 1 standard deviation of the inversion errors, computed from the frequency errors quoted by the observers assuming them to be statistically independent; the errors in the averages are correlated (Gough, Sekii, and Stark, 1996a; Howe and Thompson, 1996).

If two seismic variables are involved, as is the case for structure inversions (in the absence of rotation and a magnetic field), then two separate complementary inversions must be carried out for OLA, each requiring the suppression of the contribution to the data combination from the variable not being sought. With the help of some regularizing assumption, estimates of the seismic variables can then be made from their averages to enable one to gauge, for each seismic average, the contribution to the corresponding data combination from the other seismic variable; in most of the published "inversions", that contribution is ignored. In the case of RLS frequency fitting, the two seismic variables can be represented simultaneously.

I do not here go into how the inversions are carried out. That is not necessary for understanding the results, provided that adequate information about the averaging kernels is given. Unfortunately, that information is not always provided. Precisely how the centres of the optimally localized kernels were determined is rarely stated, although if the kernels are very narrow it doesn't much matter, unless there are large distant sidelobes. Sometimes authors use merely the locations where they tried to centre the kernels, rather than where they actually succeeded in centring them, which requires conjecture on the part of the reader relating to the information contained in the data set employed and the proficiency of the author in extracting it. One useful rule of thumb for helping to guess what might have been plotted is to recognize that with currently available frequency data well localized kernels without substantial sidelobes cannot be centred closer to the centre of the Sun than $r / R \approx 0.05$. 


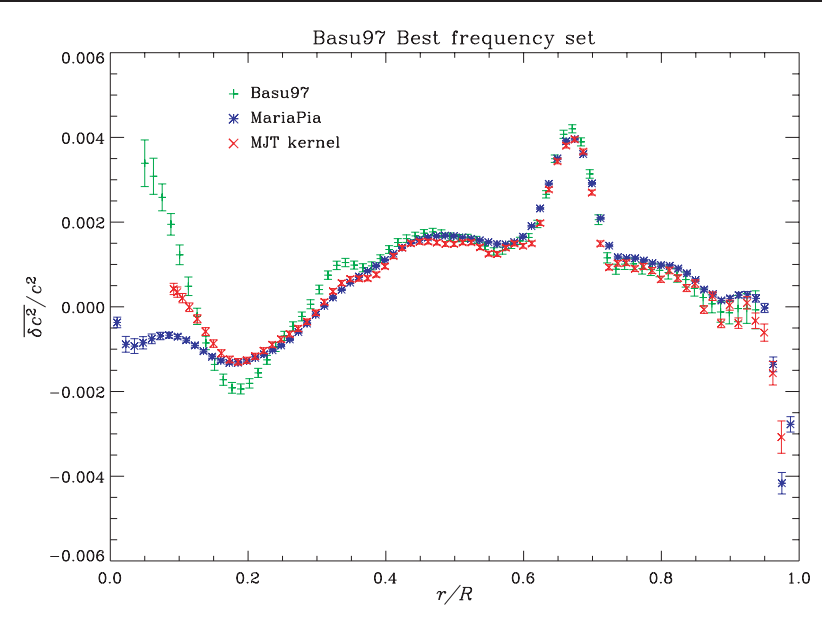

Figure 4. Several sets of optimally localized averages of the relative difference between the squared sound speed in the Sun and that in Model S of Christensen-Dalsgaard et al. (1996) computed from the same frequency data set, with kernels obtained by different inverters and by ignoring the contamination from other seismic variables. The vertical bars extend to \pm one standard deviation of the errors computed from the frequency errors quoted by the observers. [The abrupt deviation close to the surface is a characteristic of having misrepresented the acoustic radius ( $c f$. Takata and Gough, 2003).]

Inversion is not a well prescribed procedure. It is an art. And it can lead to different representations of the information, as is illustrated in Figure 4, which depicts different optimally localized sound-speed averages all derived from the same data. It should therefore be recognized that the differences between the averages plotted in the figure are not necessarily an indication of inversion error; instead, they can result from the differences in the averaging kernels selected. It is evident, therefore, that merely offering some measure of the widths of the kernels, which is absent from Figure 4, is insufficient for appreciating the results fully.

The objectives of most of our investigations do not concern seismic variables alone. I have already mentioned issues concerning the solar neutrino flux, and also the helium abundance $[Y]$ and the relation between its initial value $\left[Y_{0}\right]$ and Big-Bang nucleosynthesis. Investigation of these non-seismic quantities using seismology necessarily involves relating them to seismic variables via a theoretical model, which itself depends on the assumptions upon which that model has been built. That statement may seem obvious. But those not in our subject do not always appreciate just which of those assumptions are important. It therefore does no harm to state them. Evidently, which of the assumptions are the most important depends on the matter in hand.

I conclude this part of my discussion with another simple point, which is also not obvious to everybody: the dominant physics of helioseismology is extremely simple; it is simply the physics of the propagation and interference of well understood waves. Therefore, provided one is scrupulous in presenting the results 
with due care and attention, the direct conclusions cannot be questioned. When an observation appears to be in conflict with our seismological knowledge, as superficially seems to be the case with modern spectroscopic determinations of the photospheric heavy-element abundances, for example - an issue to which I shall turn my attention below - contrary to the opinion of some commentators (e.g. Guzik, Watson, and Cox, 2006) it is not the seismology itself that is to be challenged 4 . I hasten to add that there certainly are issues within seismology that have not been resolved, usually because a suitable way to analyse the data has not yet been found (possibly because the data do not even contain that information in a form that permits it to be readily extracted, possibly because it is impossible to do so, possibly because the extraction procedure itself still seems to be beyond our capabilities), but that is a different matter.

One further matter concerns the manner in which we use a diagnostic. That typically depends on the issue one wishes to address, and its relation to that issue may itself be subject to some doubt, perhaps due to untested assumptions in modelling. This means that the accuracy of the result may be much less than the precision, perhaps very much less. It is important to recognize the difference between the two. Precision can usually be estimated well from the precision of the data - although in order to trust the outcome one has to trust the estimated errors in the data. Accuracy requires recognizing and assessing the influence of the assumptions - inaccurate assumptions can lead to a dispersion amongst the outcomes in any investigation that prudently utilizes a variety of different analysis techniques ( $c f$. Gough, 2012a). Therefore precision is never judged to be lesser when fewer analysis procedures are considered. Unfortunately, greater precision is often mistaken for greater accuracy.

\section{Standard Solar Models}

Standard solar models are constructed usually with the most sophisticated microphysics available, yet with the most primitive macrophysics - fluid dynamical processes are ignored wherever possible, probably because they are too difficult to model in an agreed standardized manner. Rotation and magnetic fields are normally ignored too, so the star is spherically symmetrical. The star is evolved hydrostatically either from somewhere on the Hayashi track, gravitationally contracting until it reaches the main sequence, whence radiative energy loss is balanced almost exactly by nuclear energy generated in the core; or it is evolved from an estimate of the zero-age main-sequence structure, which is obtained by equating radiant energy loss with nuclear generation in a completely

\footnotetext{
${ }^{4} \mathrm{I}$ am assuming that the seismic data processers have judged their errors correctly, and that the error correlations in the frequency data sets, normally ignored, are not unduly severe. I am also assuming that, where it is appropriate, asphericity of equilibrium structure is taken correctly into account.
} 


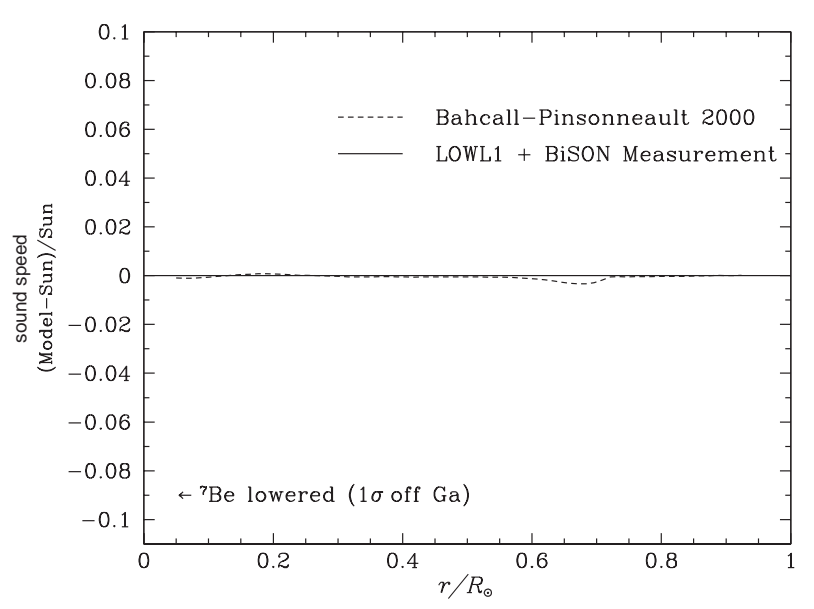

Figure 5. The dashed line is the relative difference between the sound speed in a standard solar model computed by Bahcall and Pinsonneault and that inferred by Basu (Basu et al., 1997) from a combination of oscillation frequency data obtained by Chaplin et al. (1996) and Tomczyk, Schou, and Thompson (1995a): Tomczyk et al. (1995b) (from Bahcall, Pinsonneault, and Basu, 2001). The arrow labelled ${ }^{7} \mathrm{Be}$ etc. is simply a very rough estimate of the sound-speed discrepancy in a theoretical solar model adjusted in a manner described by Bahcall, Basu, and Pinsonneault (1998) to yield a neutrino flux concomitant with that measured by the gallium detector at Gran Sasso (Hampel et al., 1996).

homogeneous star. The second approach is not quite correct, because there was some nuclear transmutation prior to arrival on the main sequence, most notably conversion of ${ }^{3} \mathrm{He}$ to ${ }^{4} \mathrm{He}$, which temporarily slows down the contraction (but is insufficient to halt it); however, that phase has little impact on the subsequent evolution of the star. Although the transition from gravitational to nuclear energy release is smooth, during subsequent evolution on the main sequence the central hydrogen abundance $\left[X_{\mathrm{c}}\right]$ declines almost linearly with time Gough, 1995), so backwards extrapolation of $X_{\mathrm{c}}$ to the initial hydrogen abundance $\left[X_{0}\right]$ provides a useful fiducial origin of main-sequence age. 
Table 1: Standard model assumptions

Initially uniform chemical composition

Spherical symmetry

Hydrostatic equilibrium

Simple description of energy transport, using mixing-length theory in convection zones, and a simplified treatment of radiative transfer in the atmosphere

No serious internal mixing of chemical species except in convection zones, so no tachocline, yet gravitational settling and chemical diffusion

Rotation dynamically negligible

Maxwell stresses negligible (i.e. no magnetism)

No mass loss

No accretion

No large-scale instability

No (nonlinear) transport by waves

Thermal balance (almost)

Equation of state known

Nuclear reaction rates (reaction cross-sections) known

Opacity formula known

Evolution on the main sequence is hydrostatic, and essentially in thermal balance, energy being transported from centre to surface by optically dense radiative transfer wherever the stratification is convectively stable: I present the transfer equation in the next section. Nuclei undergoing thermonuclear reactions are usually considered to be screened by electrons according to the classical, nonrelativistic, Debye-Hückel treatment by Salpeter (1954), although the validity of that has been questioned, most recently by Mussack and Däppen (2010, 2011). In convectively unstable regions, the transport of heat is modelled by a (usually local) mixing-length formalism; Reynolds stresses are usually, but not always, ignored. Chemical species are presumed to be homogenized in convection zones; and elsewhere, aside from nuclear transmutation, chemical differentiation is purely by gravitational settling (and possibly radiative levitation) against microscopic diffusion. Energy and material transport by the tachocline circulation is normally ignored, as is transport by acoustic and gravity waves generated by the turbulent convection. The total mass of the Sun is presumed to be conserved, and the "best" equation of state, opacity "formula" and nuclear reaction cross-sections are employed.

It goes without saying that in computing hydrostatic structure it is preferable (some would say mandatory) that the coordinate singularity at the centre 
of the star and the effective branch point at the radiative-convective interface (when a local mixing-length formalism is adopted) be correctly treated by the numerical integration, accurately enough for the resulting solar model to possess well defined seismic eigenfrequencies. Unfortunately, that is not always achieved, although I suspect that the numerical precision is greater than the observational accuracy.

\section{The Chemical Composition of the Sun}

Some time ago solar modelling (e.g. Christensen-Dalsgaard et al., 1996) had achieved fair agreement with the measured seismic structure of the Sun (e.g. Gough et al., 1996b). Indeed, Bahcall (2001) considered that to be a "triumph for the theory of stellar evolution", which he illustrated by plotting the relative deviation of the solar sound speed from that of one of his standard solar models (e.g. Bahcall, Pinsonneault, and Basu, 2001). It is reproduced here as Figure 5. Yet might one not naively consider it to be an even greater triumph for helioseismology? After all, the seismological errors are much smaller than the modelling errors, as is more clearly evident in Figures 3 and 4 , But I have already emphasized that the physics of seismology is very simple, whereas the physics of the structure of the Sun is not. The comparison can therefore be regarded in two opposite ways: i) one might marvel, as did Bahcall, that such complicated physics has been successfully reined to reproduce observation (more or less), or ii) one might consider it unremarkable that a mature theory, which has already survived a wide range of astronomical tests relating to other stars, and which depends on so many somewhat uncertain processes, can be adjusted to reproduce the measurable properties of the Sun. I think I side with Bahcall on this matter, because nobody has actually succeeded in reducing the discrepancy between the models and the Sun sufficiently to come within the much smaller seismological uncertainty. Moreover, the situation has been exacerbated by the results of recent spectroscopic re-analyses, by Asplund et al. (2009), Caffau et al. (2009, 2011) and Grevesse et al. (2011), of some of the chemical abundances in the solar atmosphere, those analyses now taking explicit account of spatial inhomogeneity in the Sun's atmosphere caused by convective motion as simulated by Asplund et al. (2000) based on a compressible hydrodynamical procedure described by Stein and Nordlund (1998) 5 . The early publications (Allende Prieto, Lambert, and Asplund, 2001, 2002; Asplund et al., 2004; Asplund, Grevesse, and Sauval, 2005a; Asplund et al., 2005b. c) suggested an enormous reduction in the total heavy-element abundance $Z$ below previously accepted values: some $40 \%$ or so.

\footnotetext{
${ }^{5}$ The original analysis employed simulations in a model atmosphere with the previously accepted chemical composition; from 2009 onwards, newer simulations were carried out with the Fe abundance inferred from the 2005 abundance determinations; the abundances of C, N, and $\mathrm{O}$ were not changed because their spectral lines are weak and have no significant impact on the radiative energy flux (R. Trampedach, personal communication, 2012).
} 
If that represents the abundance in the radiative interior, it implies, on average, a reduction in opacity of some $30 \%$. By present-day standards, that figure is enormous.

One of the best and most widely adopted solar models (combining all the generally accepted physics for so-called "standard" theory into a model computed accurately enough for reliable computations of its oscillation eigenfrequencies to be possible) is the Model S discussed by Christensen-Dalsgaard et al. (1996). It was computed with a local mixing-length theory ignoring turbulent pressure, with mixing-length parameter $[\alpha]$ and initial hydrogen and heavy-element abundances $\left[X_{0}=0.7091, Z_{0}=0.0196\right]$ chosen to yield the correct present-day radius and what was believed to be the correct luminosity, and also a resulting present surface abundance ratio $\left[Z_{\mathrm{s}} / X_{\mathrm{s}}\right]$ of 0.0245 , consistent with the spectroscopic analysis of Grevesse and Noels (1993), at an age $t_{\odot}=4.60$ Gy (notwithstanding the different value quoted by Christensen-Dalsgaard et al. (1996), and subsequently by Doğan. Bonanno, and Christensen-Dalsgaard (2010)). The individual surface abundances are $X_{\mathrm{s}}=0.7373, Z_{\mathrm{s}}=0.0181$; the present surface helium abundance is $Y_{\mathrm{s}}=0.2447$, which agrees with observation within the limits of accuracy of the equation of state and helioseismological analyses of the depression of $\gamma_{1}$ caused by the second ionization of helium in the adiabatically stratified convection zone (Gough, 1984b; Kosovichev et al., 1992: Vorontsov, Baturin, and Pamyatnykh, 1992; Basu and Antia,, 1995; Däppen, 2007), as I shall discuss briefly later. Figures 3 and 4 illustrate how well the seismic structure of the model corresponds to that of the Sun.

The heavy-element abundance now favoured by Grevesse et al. (2011) is somewhat greater than the original announcement, namely $Z_{\mathrm{s}}=0.0134 \pm 0.0005$; an independent and somewhat different analysis by Caffau et al. (2011) that also takes convective inhomogeneity into account has yielded $Z_{\mathrm{s}}=0.0153 \pm$ 0.0011. Both of these values were estimated from abundances of the major opacity-producing elements except neon, whose abundance in the Sun cannot be measured spectroscopically. A recent compilation by Lodders, Palme, and Gail (2009) using a wider range of solar data, with an eye also on meteoritic abundances, yet using simple hydrostatics, has yielded the recommendation $Z_{\mathrm{s}} / X_{\mathrm{s}}=0.0191$. Accepting $Y_{\mathrm{s}}=0.249$ from a calibration of model envelopes by Basu and Antia (2004) using seismically accessible (asymptotic) integrals that are sensitive to the depression of $\gamma_{1}$ due to He II ionization, or $Y_{\mathrm{s}}=0.224$ from a calibration of complete solar models (Houdek and Gough, 2011), yields $Z_{\mathrm{s}}=0.0141$ and $Z_{\mathrm{s}}=0.0145$, respectively. (It is unclear whether calibrating complete solar models in this fashion is more reliable or less reliable that calibrating only model envelopes.) These values are all substantially lower than that of Model S, on average by about $20 \%$. What are the implications of these results? 
5.1. The Abundances in Context

Many of the discussions that have ensued have carried out solar-evolution computations and then compared, in some manner, implied seismic data, or seismic structure (principally sound speed), with the inferences from the Sun. They have been catalogued by Basu and Antia (2008). However, seismic structure is merely a diagnostic of the issue, not the issue itself. It is instead much more lucid to the average physicist to be confronted with the direct implication of the newly reported abundances. So let us accept (most of) the assumptions of solar modelling, as is usually done in the discussions of the problem, and ask what they really imply. I start with the most obvious: that the Sun approached the main sequence fully mixed, having just come down the Hayashi track. Therefore the heavy-element abundance $\left[Z\left(r, t_{\odot}\right)\right]$ in the radiative interior today is (almost) the same as it is in the photosphere, aside from a relative excess of $3 \%$ or so due to differential gravitational settling. Heavy elements influence the structure of the Sun primarily via their effect on the opacity $[\kappa]$, which controls the relation between luminosity $[L]$ and temperature $[T]$ through the equation of radiative transfer: $L(r, t)=-\left(16 \pi a c r^{2} T^{3} / 3 \kappa \rho\right) \mathrm{d} T / \mathrm{d} r$. However, $T$ is not a seismic variable, and therefore cannot be measured directly. It is related to the seismic variables $c^{2}$ and $\rho$ by the equation of state: $T=\mathcal{T}\left(c^{2}, \rho ; Y, Z\right)$ - I set aside, for the moment, consideration of the relative abundances of the heavy elements. The contribution of $Z$ to the equation of state is only about $0.5 \%$, so for the purposes of this discussion its uncertainty can safely be ignored. There remains only the helium abundance $[Y]$ which is to be estimated from the theory of stellar evolution. It is important to appreciate that this is the only stage in the argument where the details of the evolution theory come into serious play. However, it must be realized that knowledge of the functional form of $Y(r)$ today is crucial for assessing the stratification of the Sun's radiative interior. Therefore, some attention must be paid to how it is determined, appreciating the assumptions to which the outcome is sensitive.

As the Sun evolves on the main sequence, hydrogen is transmuted into helium in the core, adding a spatially and temporally varying component $\delta_{\varepsilon} Y$ to the helium abundance: $Y(r, t)=\left[1+s_{Y}(r, t)\right] Y_{0}+\delta_{\varepsilon} Y$, where $s_{Y} Y_{0}$ is the change in $Y$ produced by gravitational settling moderated by diffusion. It is known (e.g. Gough, 1983c, 1990) that the time dependence of the total luminosity $L_{\mathrm{s}}(t):=L(R, t)$ of the solar model, calibrated to satisfy $L_{\mathrm{s}}\left(t_{\odot}\right)=L_{\odot}$, is not very sensitive to the details of the theory (such as the choice of $Z_{0}$, or whether or not there has been some small degree of material mixing in the core - we know from seismology that the core cannot have been homogenized, a conclusion which is consistent with HR diagrams of solar-like stars), so the total amount of helium that has been produced by today $\left[\int \delta_{\varepsilon} Y \mathrm{~d} r\right]$ is proportional to $\int L_{\mathrm{s}} \mathrm{d} t$, which is essentially known (provided $t_{\odot}$ is known). Therefore it is adequate for the current discussion to accept the function $\delta_{\varepsilon} Y$ from any standard model. Also, the function $s_{Y}(r, t)$ is only very weakly dependent on $Y_{0}$, and may also safely be taken as given by the model. Likewise, one can take $Z=\left[1+s_{Z}(r, t)\right] Z_{0}$ 
with $s_{Z}$ given by the model (it is broadly similar to the function $s_{Y}$ ). Whence $T(r, t)=\mathcal{T}\left(c^{2}, \rho ;\left(1+s_{Y}\right) Y_{0}+\delta_{\varepsilon} Y,\left(1+s_{Z}\right) Z_{0}\right)$, where $c^{2}$ and $\rho$ have the values determined seismologically from the Sun. Only the initial abundance $Y_{0}$ now remains unknown. How do we determine it?

I recommend calibrating $Y_{0}$ by accepting the nuclear reaction rates - after all, all the pertinent nuclear cross-sections have been carefully assessed experimentally during the investigations of the neutrino problem, save the controlling $\mathrm{p}-\mathrm{p}$ reaction cross-section which is determined only theoretically (however, it is the simplest of all nuclear reactions). There remains also an issue concerning the screening of energetic particles (e.g. Mussack and Däppen, 2010, 2011). These matters are probably, for the purposes of this discussion, minor. Therefore the total rate of thermal energy production, hence the luminosity, is determined in terms of $t_{\odot}$ and $Y_{0}$. Granted that $L_{\odot}$ and $t_{\odot}$ are known quite well, $Y_{0}$ can thus be calibrated to determine $T(r)$ today 6 , which can then be substituted into the equation of radiative transfer to evaluate $\kappa(r)$. That procedure can either be carried out explicitly from a seismological inversion to determine the seismic stratification, or implicitly within the inversion itself, such as in the manner advocated by Elliott (1995). The outcome is illustrated in Figure 6, in which the continuous curve is the relative difference $\left(\kappa_{\odot}-\kappa_{\mathrm{S}}\right) / \kappa_{\mathrm{S}}$ between the Sun's opacity $\left[\kappa_{\odot}\right]$ determined in the manner that I have just described and that of Christensen-Dalsgaard's Model S. Not surprisingly, $\kappa_{\odot}$ is very similar to the opacity in Model S. The issue posed by Asplund et al. and Caffau et al. is

${ }^{6}$ Implications from earlier estimates of $Y$ to determine $T(r)$ have been discussed by Elliott (1995) and Tripathy and Christensen-Dalsgaard (1998), the first under the assumption that $Y(r)$ differs from that in a reference solar model by just a constant, the second that it can be obtained simply by scaling the reference-model value by a constant factor, both of which are inaccurate in the core, although Elliott notes that the opacity perturbations produce a predominantly local response, so that these analyses should provide a fair estimate in the radiative envelope. The Tripathy-Christensen-Dalsgaard scaling was used by Tripathy, Basu, and Christensen-Dalsgaard (1998) to obtain the opacity difference from Model S by RLS frequency fitting (J. Christensen-Dalsgaard, personal communication, 2012) assuming that that difference can be expressed as a function of $T$ alone, yielding a superficially similar functional form to the continuous curve in Figure 6 , but with a magnitude about 50 per cent greater. Bahcall et al. (2005) have estimated the opacity difference by adjusting $\kappa$ by hand in solar models. Their preferred model had a constant $11 \%$ augmentation over the OPAL values using the most recent abundance determinations (Asplund et al. . 2000. 2004: Asplund. 2005: Allende Prieto, Lambert, and Asplund, 2001, 2002, Asplund personal communication with Bahcall et al., 2004) in the radiative envelope down to $T=5 \times 10^{6} \mathrm{~K}$, beneath which the augmentation was smoothly reduced to zero (probably by a half Lorentzian function with half-width at half maximum of $2 \times 10^{5} \mathrm{~K}$ ), and has a seismic structure as close to that of the Sun as does Model S. Regarding that model as a proxy Sun, one would expect the relative opacity difference between it and a model with the unmodified abundances to be comparable with the inference by Christensen-Dalsgaard et al. (2009). The two estimates are depicted in Figure 6. Korzennik and Ulrich (1989) had earlier estimated opacity errors by RLS (L2 norm) data fitting, and Saio (1992) by $\mathrm{L}_{1}$ data fitting, each by scaling $\kappa$ by a function of $T$ and ignoring the dependence of the relation between $T$ and the seismic variables on chemical composition; they expressed their results as deviations from different reference models, so they cannot easily be compared with those presented in Figure 6. An estimate by OLA (Takata and Gough, 2001) of the absolute structure of the Sun, including opacity, using the procedure for determining $Y$ described in the text (together with tachocline homogenization as calibrated by Elliott. Gough, and Sekii (1998)) is presented by Gough and Scherrer (2002) and Gough (2004, 2006). 


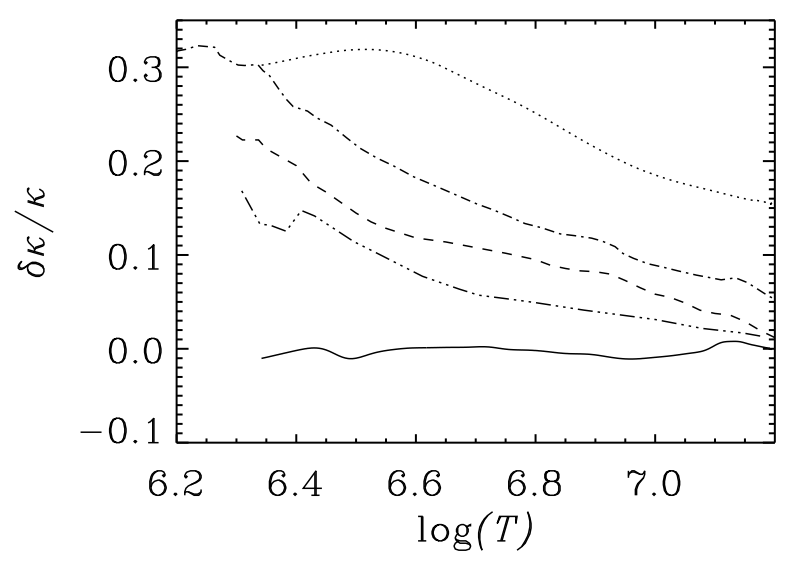

Figure 6. The dot-dashed curve is the estimate by Christensen-Dalsgaard et al. (2009) of the relative opacity augmentation required when adopting the Asplund (2005) chemical composition in OPAL opacity computations (Iglesias and Rogers, 1996) to produce a solar model with the same sound speed as in Model S of Christensen-Dalsgaard et al. (1996). The dashed curve represents the augmentation, computed by Christensen-Dalsgaard and Houdek (2010), required of OPAL opacities with the revised Asplund et al. (2009) abundances. The triple-dot-dashed curve is a comparable difference between two solar models computed by Bahcall et al. (2005) with OPAL opacities, the first with the latest abundances by Asplund et al. at the time, the second with abundances that have been artificially chosen to yield what one might regard as a seismically acceptable proxy Sun. For comparison, the dotted curve is a linearized estimate of the same quantity were the relative abundances of the heavy elements to have been preserved, using opacity derivatives obtained from the tables of Bahcall and Pinsonneault (1992)). The continuous curve is the relative difference $\left(\kappa_{\odot}-\kappa_{\mathrm{S}}\right) / \kappa_{\mathrm{S}}$ inferred by Gough (2004) between the Sun's opacity $[\kappa \odot]$ and that of Model S.

therefore simply an abundance-opacity problem: how can $\kappa_{\odot}(r)$ be reconciled with their abundance measurements?

\subsection{Suggestions for Reconciliation}

An obvious naive suggestion is that opacity calculations are in error by just the appropriate factor required to compensate for the proposed revision in $Z$, leaving the functional dependence on the other variables unscathed. Given the testing that has been undertaken by Iglesias and Rogers, that does not seem so very likely. It is also unlikely that energy transport is opposed by some other mechanism, such as gravity-wave transport, because that would require a completely different physical process to mimic the functional form of the effect of radiatively induced atomic transitions. Of course, it could be that the new abundance determinations are in error, despite the extra care that has been taken; we have seen an increase since the earlv work by Allende Prieto, Lambert, and Asplund (2001, 2002) and Asplund et al. (2004); Asplund, Grevesse, and 
Sauval (2005a); Asplund et al. (2005b, C), so a further increase might not be too surprising. However, the independent investigation by Caffau et al. (2009, 2011) has yielded results not very dissimilar from those of Asplund et al. (2009) and Grevesse et al. (2011), which adds to the credibility of the new abundances. Of course, another possibility is that early in its post-Hayashi days the Sun was contaminated by metal-poor material, as Guzik. Watson, and Cox (2005) have suggested. If that were the case, it may just be possible to detect seismologically a relic compositional discontinuity at the location of the base of the zero-age convection zone, provided that it has not been destroyed. The discontinuity is at least stable to double-diffusive convection, so it would not be destroyed spontaneously. Yet a fourth possibility is that the relative abundances of the heavy elements are different from what is generally believed, particularly that of neon, as has been discussed, for example, by Drake and Testa (2005), leaving open the possibility that $Z$ is greater than the values suggested by Asplund et al. and Caffau et al. based on the assumption that the relation of the neon abundance to the abundances of the other opacity-producing elements is preserved, and that therefore $\kappa$ is perhaps proportionately greater too. That too is regarded by many spectral analysts as being intrinsically unlikely, and in any case neon alone does not mimic the heavy-element mixture adequately to restore the solar models to their pre-Asplund state.

The apparent opacity discrepancy has evolved with the abundance revisions. Christensen-Dalsgaard et al. (2009) estimated the amount $\delta \kappa$ by which the opacity according to OPAL (Iglesias and Rogers, 1996) with Asplund (2005) chemical composition would need to be augmented in order to produce an otherwise standard solar model with the same sound speed as in Model S, and hence about the same sound speed as in the Sun. It is depicted as the dot-dashed curve in Figure [6] where it is plotted against $\log (T)$ along the thermodynamic $\rho-T$ path of one of the models. One might have expected the outcome to have been given approximately by $(\partial \ln \kappa / \partial \ln Z)_{\rho, T, X} \delta \ln Z$, in which $\delta \ln Z \approx \ln Z_{\mathrm{S}}-\ln Z_{\mathrm{Asp}} \approx \ln Z_{\mathrm{s}, \mathrm{S}}-\ln Z_{\mathrm{s}, \mathrm{Asp}}=0.370$, the subscript $\mathrm{s}$ denoting surface value and the subscripts $S$ and Asp denoting Model S (Grevesse and Noels, 1993) and Asplund (2005) values respectively, and where the opacity derivative is evaluated at constant relative heavy-element abundances. However, it is evident from Figure [6 that that is not the case. According to Jørgen Christensen-Dalsgaard (personal communication, 2011) the quite substantial difference arises because the relative heavy-element abundances in the two models differ. Unfortunately, that renders back-of-the-envelope estimates suspect. The later abundance determinations by Asplund et al. (2009) have brought the OPAL opacities closer to those of the Sun, as the dashed curve in Figure 6 depicts. It is interesting that Antia and Basu (2011), using the chemical composition proposed by Caffau et al. (2011), whose relative abundances do differ from the earlier values given by Grevesse and Sauval (1998), and Grevesse and Noels (1993), and presumably also the more modern values of Grevesse et al. (2011), report that they have obtained solar models that are seismically almost as good as models constructed with the abundances of Grevesse and Sauval (1998), and they found even closer agreement with the Sun if the assumed abundance of neon 
were artificially enhanced by a factor $\sqrt{2}$. That essentially reduces the problem of reproducing the Sun's seismic stratification theoretically to the state in which it was prior to Asplund's original announcement. It must be realized, however, that that does not close the matter, because merely reproducing previous partial results does not necessarily prove the veracity of the physics behind the new models. Moreover, the seismic structures of the models, new and old, deviate from that of the Sun by many standard errors, as is evinced by Figure 3

\subsection{Possible Flaws in the Argument}

The foregoing discussion is predicated on the presumption that the sole significantly discrepant ingredient in the solar modelling is opacity. That need not be the case; modification of any non-seismic variable might, at least in principle, bring a theoretical model into line with observation. Evidently, a direct change in the relation between the seismic variables pressure and density, for example, via a modification to the abundances of the abundant chemical elements $\mathrm{H}$ and $\mathrm{He}$, can alter the sound speed: Gough and Kosovichev (1988, 1990) found that the stratification of the energy-generating core can be reproduced by a slight smoothing of the abundance profile that had been produced by nuclear transmutation, suggesting that a slight degree of mixing has taken place, possibly by disturbances that have been shear-generated in a manner analogous to clear-air turbulence in the Earth's atmosphere. Alternatively, there could be an additional source of energy transport without mixing, such as by accreted weakly interacting massive particles (wimps), whose presence in the Sun was postulated originally in order to try to account for the observed low neutrino flux without neutrino transitions (Spergel and Press, 1985); wimps modify the temperature distribution, and consequently the distribution of helium produced by nuclear transmutation, leading to a sound-speed modification whose general functional form is broadly similar to what would be required (Gilliland et al., 1986), although a serious attempt to reproduce the seismic structure of the radiative interior appears not to have been made (see also Faulkner, Gough, and Vahia, 1986; Däppen, Gilliland, and Christensen-Dalsgaard, 1986; Christensen-Dalsgaard, 1992).

It is worth commenting that a reduction in $Z_{\mathrm{s}}$ at fixed relative abundances of only about the $20 \%$ below that of Model S implies, via the theory of solar evolution, a reduction of about 0.02 in $Y_{\mathrm{s}}$. This appears to me to be not dissimilar to the margins of the true uncertainty in the helioseismological determinations of the helium abundance of the convection zone - although not within the precision quoted by those who have attempted to determine $Y_{\mathrm{S}}$ by only a single procedure (e.g. Basu and Antia, 1995, 2004: Basu, 1998; Richard et al., 1998: Di Mauro et al., 2002), even granted that systematic errors had been recognized. 
5.4. Seismological Investigation

Chemical abundances can in principle be measured directly by seismology by analysing the non-ideal properties of the solar plasma. Specifically, $\gamma_{1}$ is depressed in regions of partial ionization, by an amount that is almost proportional to the abundance of the ionizing element. Däppen and Gough (1984) proposed measuring the helium abundance by calibrating the ionization-induced variation of a thermodynamic function $[\Theta]$ expressible in terms of $\gamma_{1}$ and its derivatives and which is easily accessible to seismological probing in the adiabatically stratified layers of the convection zone (Gough, 1984b). A procedure for so doing was developed by Däppen. Gough, and Thompson (1988), who found $Y_{\mathrm{s}}$ to be lower than typical values obtained from calibrating evolved solar models (Däppen et al., 1991), which triggered Christensen-Dalsgaard. Proffitt, and Thompson (1993) to investigate the seismological implications of gravitational settling of heavy elements, with notable success. Other forms of calibration, often based more directly on $\gamma_{1}$, have been pursued (e.g. Däppen et al., 1991; Basu and Antia, 1995, 2004: Basu. 1998: Richard et al.. 1998: Di Mauro et al.. 2002: Houdek and Gough, 2007). None has been completely satisfactory, not least because there are serious uncertainties in the equation of state. There have been attempts to estimate $Z$ by calibrating solar models against low-degree frequency-separation ratios assuming $t_{\odot}$ to be known (e.g. Chaplin et al., 2007); that is equivalent to calibrating $Y$, of course.

A direct seismological estimate of $Z$ in the convection zone, and thus a direct estimate of $Z_{\mathrm{s}}$, via the ionization-induced depressions in $\gamma_{1}$ or in the variation of the seismologically more accessible function $\Theta$ of $\gamma_{1}$ and its derivatives, is very delicate. It would probably be necessary to average the individual variations in the different ionization zones, so the result would depend to some degree on the assumed values of the relative abundances (Mussack and Gough, 2009). Nevertheless, if successful, the accuracy of the result could probably be assessed from the seismic frequency uncertainties, because the relation between the magnitude of the $\gamma_{1}$ depression and the abundance of any ionizing element producing it, at least at the level required to judge the abundance-opacity problem, is fairly robust. The seismologically apparently more straightforward procedure of measuring a spatially averaged absolute value of $\Theta$ and relating that to the slightly greater value expected in the absence of heavy elements. as Antia and Basu (2006) have tried, is more uncertain because the change in $\Theta$ due to heavyelement ionization that is sought appears to be less than the uncertainty in the equation of state arising, for example, from the commonly neglected, at least in the chemical picture (Däppen, 2004, 2007), finite volume occupied by bound species (e.g. Baturin et al., 2000). 
5.5. The Equation of State

My statements about the uncertainty in $Y_{\mathrm{s}}$ and the equation of state call for some justification. It goes without saying that chemical composition is not a seismic variable, not even the abundances $X$ and $Y$ of the abundant elements $\mathrm{H}$ and He. In order to determine $Y$, say, it is evidently necessary to use an equation of state, which relates the seismic variables to composition via another, necessarily non-seismic, variable, such as $T$ or $s$, and subsequently, at some point, to confine attention to the adiabatically stratified region of the convection zone where that variable can be eliminated from the spatial variation of the seismic variables. Once that has been accomplished one can learn about chemical composition through the effect of ionization on $\gamma_{1}$. This has been approached either directly by measuring some appropriate property of $\gamma_{1}$ itself, or by working with some thermodynamic function $\Theta$ of it. The reliability of the outcome necessarily rests on the reliability of the equation of state, which is very difficult to assess. That should be obvious because there is no redundancy in the dependence of the seismic modes on the seismic variables. Therefore, as has been alluded to in the past (e.g. Gough, 2004), perhaps too obliquely, any intrinsic error in the equation of state cannot be assessed by seismology alone. To make progress, non-seismic information must be incorporated. In practice, that information comes from some prior appreciation of the reliability of some aspects of the equation of state, coupled with the additional non-seismic constraint that the convection zone is chemically homogeneous (sometimes augmented with the constraint that deep down the stratification is adiabatic). Suppose, for example, one is estimating the deviation of the Sun from a reference solar model, represented by $\delta \ln \gamma_{1}$ and $\delta \ln u$, where $u(r)$ is a complementary seismic variable. From seismology one can relate averages $\overline{\delta \ln \gamma_{1}}+\overline{\overline{\delta \ln u}}$ of those deviations to data combinations $d$, where the single and double overbars indicate simply that the averaging kernels are different. Then, one can write the first average as the sum of $\overline{\left(\partial \ln \gamma_{1} / \partial \ln u\right)_{Y} \delta \ln u}$, which can be incorporated into the second average, of $\overline{\left(\partial \ln \gamma_{1} / \partial Y\right)_{u}} \delta Y$, where $\delta Y$ is the difference in $Y$ between the Sun and the reference model, and of a component $\overline{\delta_{\text {int }} \ln \gamma_{1}}$ resulting from intrinsic error in the equation of state. One would like to be able to distinguish between those components. However, that is strictly impossible because they have the same averaging kernel. Therefore one cannot unambiguously determine $\delta Y$ from the data $[d]$. What has been attempted in the past is to design kernels such that those defining the overbar and the double overbar are both in some sense small, and then to estimate $\delta Y$ by neglecting $\overline{\delta_{\text {int }} \ln \gamma_{1}}$ and $\overline{\overline{\delta \operatorname{lnu}}}$, a procedure which is evidently not strictly valid; after that, $\overline{\delta_{\text {int }} \ln \gamma_{1}}$ can be computed with presumably different and well localized averaging kernels. The results of such a procedure have been presented by Basu. Däppen, and Nayfonov (1999), Di Mauro et al. (2002), and Däppen (2004), using both OPAL and MHD, the two most popular, and probably the best, equations of state available today. Not only do their inferred intrinsic errors from the two equations differ, as they surely must, but so too do the estimates of the helium abundance $\left[Y_{\mathrm{S}}\right]$ in the Sun's 
convection zon 7 . It must be appreciated that any seismological inference about $\delta_{\text {int }} \ln \gamma_{1}$ such as this is susceptible to potential errors in the inferred values of $Y_{\mathrm{s}}$, which are difficult to appreciate because the precise manner in which $\delta Y$ was obtained is unclear. However, well outside the hydrogen and helium ionization zones $\partial \ln \gamma_{1} / \partial Y$ is small, so errors in $\delta Y$ can hardly contaminate $\overline{\delta_{\text {int }} \ln \gamma_{1}}$ there. This is a robust property of any realistic equation of state, because under normal stellar conditions $\gamma_{1}$ is essentially independent of $Y$ where hydrogen and helium are both fully ionized, errors in the small dependency that does remain (arising from processes such as the influence of the electron density on the ionization of heavy elements) being characterized by the quantity $\overline{\delta_{\text {int }} \ln \gamma_{1}}$ itself. However, the situation is different within the ionization zones, where in general $\overline{\delta_{\text {int }} \ln \gamma_{1}}$ and $\overline{\left(\partial \ln \gamma_{1} / \partial Y\right)} \delta Y$ contribute comparably, and cannot be entirely recognized apart. I must add, however, that $\overline{\delta \ln \gamma_{1}}$ and $\overline{\left(\partial \ln \gamma_{1} / \partial Y\right)}$ are normally functionally different, so $\overline{\delta_{\text {int }} \ln \gamma_{1}}$ cannot vanish everywhere.

An alternative, more transparent, approach to an attempt at separation could be to adopt the attitude that one's chosen equation of state is as good as it can be, at least in the adiabatically stratified region where $\overline{\delta \ln \gamma_{1}}$ has been inferred. Therefore one could choose for each equation of state the value of $\delta Y$ that minimizes the integral with respect to acoustic radius of $\left(\delta_{\mathrm{int}} \ln \gamma_{1}\right)^{2}$ over that region - I choose acoustic radius because that is the natural seismic frequencycontrolling independent variable. The outcome, using the inferences of $\overline{\delta_{\text {int }} \ln \gamma_{1}}$ and $Y_{\mathrm{s}}$ by Di Mauro et al. (2002), is $Y_{\mathrm{s}}=0.2430$ for OPAL and $Y_{\mathrm{s}}=0.2280$ for MHD; I do not quote a precision because it is hardly material to estimating accuracy in this context. The range of all these values, roughly 0.02 , must surely be regarded as a lower bound to the range of values within which the solar $Y_{\mathrm{s}}$ is likely to lie, because the discrepancies are systematic; that value is some $40-$ 100 times greater than typical errors quoted by, for example, Basu (1998) or Di Mauro et al. (2002), based on the precision of the specific procedures that were carried out. Based solely on the calibrations described in this paragraph, one might optimistically conclude that $Y_{\mathrm{s}}=0.24 \pm 0.01$, while recognizing that the accuracy might well have been overestimated. Consequent estimates of $\overline{\delta_{\text {int }} \ln \gamma_{1}}$ are illustrated in Figure 7

${ }^{7}$ Di Mauro et al. quote $Y_{\mathrm{s}}=0.2539 \pm 0.0005$ when OPAL is used, $Y_{\mathrm{S}}=0.2457 \pm 0.0005$ when MHD is used, the errors being merely formal, representing a precision error that takes no account of the error in the relation between $Y_{\mathrm{S}}$ and $\gamma_{1}$ in the reference model. With those values the magnitude of the inferred $\overline{\delta_{\text {int }} \ln \gamma_{1}}$ was found to be the greater for the MHD equation of state beneath the helium ionization zones, and the lesser above $r / R \approx 0.97$ in the He II ionization zone. That is perhaps not surprising because MHD is possibly better at taking into account the complicated chemistry that dominates higher up in the solar envelope, whereas the virial expansion used for OPAL is perhaps more reliable where such complications make only a minor non-ideal contribution. The difference between OPAL and MHD must surely offer some estimate of the total uncertainty. 


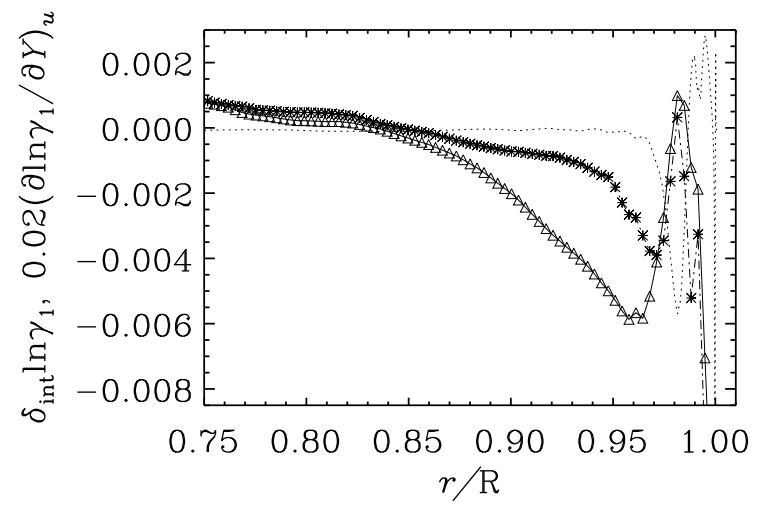

Figure 7. Inferences concerning the intrinsic error in the OPAL (asterisks) and MHD (triangles) equations of state, based on the assumption that the helium abundance, in each case, is such as to minimize its $\mathrm{L}_{2}$ norm with respect to acoustic radius over the region of the convection zone that is stratified adiabatically to better than 1 part in $10^{3}$, computed from the analysis of Di Mauro et al. (2002). Because the helium abundance is uncertain, the symbols should not be meant to represent reliable estimates of the actual intrinsic errors $\overline{\delta_{\text {int }} \ln \gamma_{1}}$. Formal error bars are not drawn because they are enormously smaller than the true uncertainties, and might therefore confuse. The dotted curve is $0.02\left(\partial \ln \gamma_{1} / \partial Y\right)_{u}$, computed with the OPAL equation of state.

\subsection{Other Matters to Consider}

There are uncertainties in other quantities used in estimations of the chemical composition that still need to be addressed. Amongst them are the current luminosity $\left[L_{\odot}\right]$ and the age $\left[t_{\odot}\right]$, a matter to which I have already alluded. The luminosity is inferred from the total solar irradiance, and is currently obtained by assuming that the radiant output is spherically symmetric (e.q. Willson and Hudson, 1988). That assumption is not strictly correct, as models of solarcycle irradiance variation indicate (e.g. Tavlor et al., 1998; Foukal et al., 2006; Fröhlich, 2011); accounting for the asphericity augments the estimate of $L_{\odot}$ by some $1.5 \%$ or so. However, there has been a recent downward revision in the measured value of the irradiance from an end-to-end instrumental recalibration (Kopp and Lean, 2011), which is partially compensating. The age [ $\left.t_{\odot}\right]$ is more uncertain. It is likely to be about the same or only slightly greater than the age of the oldest meteorites, which seem to lie between 4.563 and $4.576 \mathrm{Gr}$ (Amelin et al., 2002; Jacobsen et al., 2008, 2009; Bouvier and Wadhwa, 2010). Attempts to calibrate solar models seismically have yielded values equal to or slightly lower than the age $t_{\mathrm{S}}$ adopted for Christensen-Dalsgaard's model S, namely 4.60 Gy. Those calibrations in which a value of $Z_{\mathrm{s}}$ is assumed to be that of Model S yield a value rather lower than $t_{S}$ (Dziembowski et al., 1999: Bonanno, Schlattl, and Paternò, 2002; Doğan, Bonanno, and Christensen-Dalsgaard, 2010), the most recent, based on BiSON data (Chaplin et al., 2007) being $4.57 \mathrm{~Gy}$; 
a simultaneous calibration of $Z_{\mathrm{s}}$ and $t_{\odot}$ by Houdek and Gough (2011), also with BiSON data (Basu et al., 2007), has yielded precisely $t_{\mathrm{S}}$. Interestingly, the heavy-element abundance in the latter investigation was calibrated to be $Z_{\mathrm{s}}=0.0142$, essentially the same as the recent value preferred by Lodders, Palme, and Gail (2009), and lying between the values preferred by Asplund et al. (2009) and Caffau et al. (2011). However, the model was calculated with the relative heavy-element abundances of Grevesse and Noels (1993) (neon not being artificially enhanced); therefore the opacity is too low and the seismic structure cannot be correct8. Whether that has yielded a superior or an inferior estimate of $t_{\odot}$ is unclear (Gough, 2012a). The calibration relies partially on estimating $Y_{\mathrm{s}}$ seismologically from the oscillatory component of the low-degree frequency distribution caused by the acoustic glitch associated with the depression of $\gamma_{1}$ in the helium ionization zones (Houdek and Gough, 2007). To accomplish that estimate it was assumed that hydrostatic support is solely a balance between pressure gradient and gravity, as is usual: magnetic stresses and the centrifugal force were ignored. (It was also assumed that the Sun is spherically symmetric, as in other calibrations, which we know is not strictly correct (e.g. Goode et al., 2002; Komm. Howe, and Hill, 2006; Emilio et al., 2007; Fivian et al., 2008; Kuhn et al., 2012); by how much the structural asymmetry contaminates the assumed relation between the degree-dependence of the seismic frequencies and the locations of their lower turning points, of crucial importance to the structural calibrations, is yet to be ascertained.)

The validity of ignoring magnetic stress has been implicitly questioned by Basu and Mandel (2004) and Verner. Chaplin. and Elsworth (2006), who reported a $10 \%$ solar-cycle variation in the glitch signal. That variation is huge, and, if correct, cannot possibly result from a temporal abundance variation. It has been pointed out that were the glitch variation to be magnetic, an intensity variation of some ten or so Tesla in the second He ionization zone would be implied (Gough, 2006). Moreover, it would introduce an error in the two-parameter age calibration of similar relative magnitude (Houdek and Gough, 2011). However, Christensen-Dalsgaard et al. (2011) have recently failed to detect a change of such magnitude, although they admit that further analysis is necessary in order to be sure. Meanwhile, Basu et al. (2010) maintain that the change does occur. The whole issue is evidently very important, and we would like to know the answer. It may be some time before we do.

\footnotetext{
${ }^{8}$ Moreover, the initial helium abundance $\left[Y_{0}\right]$ of the calibrated model is about 0.250 , which is dangerously close to the amount $\left[Y_{\mathrm{p}}\right]$ believed to have been created by Big-Bang nucleosynthesis, whose estimated value has been climbing over the last two decades (Steigman, 2007): the latest estimate is $Y_{\mathrm{p}}=0.2478 \pm 0.0006$ (G. Steigman and M. Pettini, personal communication, 2011). Subsequent contamination of the interstellar medium by supernovae exacerbates the situation.
} 


\section{What of Importance is There Left to be Learnt?}

Helioseismology was amazingly successful in the early days. It demonstrated that the resolution of the solar neutrino problem was not to be found in adjustments to parameters of a standard solar model, and later, as precision increased, that the resolution must be sought in nuclear or particle physics. As we all now know, the matter is resolved by neutrino transitions. Helioseismology has also provided estimates of the solar helium abundance in the convection zone, perhaps not as accurately as we would like, yet which, granted gravitational settling, are at least not seriously at variance with Big-Bang nucleosynthesis. Coupled with that is a precise measure of the location of the base of the convection zone (Christensen-Dalsgaard, Gough, and Thompson, 1991; Basu and Antia, 1997), which has been used extensively as a simple diagnostic for calibrating theoretical solar models (e.g. Turck-Chièze et al., 1993; Bahcall. Pinsonneault, and Basu, 2001; Guzik. Watson, and Cox, 2005), and is important for defining the boundary conditions in numerical simulations of the convection zone. In addition, we know that the quadrupole moment $\left[J_{2}\right]$ of the exterior gravitational equiptentials is about $2.2 \times 10^{-7}$ (e.g. Schou et al., 1998; Antia. Chitre, and Gough, 2008), contributing to the precession of planetary orbits, particularly that of Mercury, by an amount that is compatible with General Relativity. The latitudinal variation of the angular velocity $[\Omega]$ in the photosphere persists, approximately, to the base of the convection zone, beneath which is a thin interface, the tachocline, and a rigidly rotating interior (admittedly with some doubt about the rotation of the energy-generating core).

We seem to have answered the major outstanding questions accessible to seismology that interest most of the scientific community. So is global helioseismology finished? Is helioseismology finished? To be sure, there is the unfinished business I talked about in the previous section, but it may require only minor details to sort that out. It is now often suggested by commentators that the days of true excitement in the subject, and of important discovery, are over.

I dissent vehemently from that view. Firstly, the issues that I discussed in the previous section are related to the microphysics of opacity and the equation of state, which are important to plasma physics and may end up being very important also to the study of stars other than the Sun. More detailed seismological investigation may be able to unveil, or at least provide clues to, some fundamental unresolved issues in plasma physics, such as, in the chemical picture, how the energy difference between the continuum of "unbound" electrons and the strongly bound states is determined. This is related to the whole matter of electron screening of charged species, a reliable consistent quantum-mechanical study of which is still wanting, and has implications also for thermonuclear reaction rates (e.g. Däppen, 1998). But more obvious is the whole matter of the internal macroscopic dynamics of the Sun: large- and medium-scale angularmomentum transport; material redistribution by meridional flow in the radiative zone; the pattern of the large-scale meridional flow in the convection zone; 
augmentation, distortion, and decay of the magnetic field and its back-reaction on the flow; the formation, evolution, and final decay of sunspots and other forms of activity; and how all these conspire to drive and control the solar cycle, and, perhaps most prominently, modulate the solar outputs of particles and electromagnetic radiation that influence the climate on Earth. Many of these are the object of ongoing local helioseismological techniques - time-distance and seismic holography, and ring analysis. I have not discussed those techniques here. Ring analysis is relatively straightforward, and has given us views of horizontal flow not far beneath the photosphere. Telechronoseismology has given us a view of a sunspot, complete with the flow around it: deep divergent horizontal flow carrying away the excess heat rising around the obstructing spot and an associated convergent subphotospheric counterflow above (Zhao, Kosovichev, and Duvall, 2001: Zhao and Kosovichev., 2003: Kosovichev. 2009: Zhao, Kosovichev, and Sekii, 2010). Divergent (Evershed) motion has now been detected even closer to the photosphere (Zhao et al., 2011) - a critical feature that was missing from the original seismological analysis, and which caused some onlookers to harbour grave doubts. The qualitative picture, which has made its way onto the wellknown SOI/MDI coffee mug, is now not only physically plausible, but also in accord with photospheric observations. A dynamical picture of the Evershed flow is now emerging (e.g. Weiss et al., 2004; Kitiashvili et al., 2009, 2010). More recent helioseismic observations (Kosovichev and Duvall, 2011) have revealed the cessation of the deeper subphotospheric convergent flows as sunspots decay, flows which one might presume had previously held the spots intact. However, different seismological analyses are not vet all entirely consistent (e.q. Gizon et al., 2009; Hindman, Haber, and Toomre, 2009), so the picture is not wholly secure. Also, more extensive observations will be needed for understanding the entire life-cycle of the spots.

Another matter of importance for understanding the global internal dynamics is the structure of the tachocline and its associated meridional flow, and how that influences the convection above. It is extremely difficult to detect deep meridional flow seismologically, because the frequency perturbations are small; however, some progress appears to be possible with the use of eigenfunction distortions (Schou. Woodard, and Birch, 2009; Gough and Hindman, 2010), and Antia, Chitre, and Gough (2012) are trying to advance simplified seismologically constrained dynamical arguments. If the meridional tachocline flow is downwelling near the Equator and the Poles, and upwelling near the latitude of zero tachocline shear, as Spiegel and Zahn (1992) and Gough and McIntyre (1998) have argued, how does that flow connect to the general meridional flow in the convection zone? Or is that flow so slow that it is simply swept aside unnoticed by an independent Reynolds-stress-driven circulation? Does the upwelling dredge up a primordial magnetic field from beneath, and if so, does the shearing of that field in the tachocline react back on the rotation to produce a mid-latitude shear-free region? Does the dredged field stoke a (non)dynamo in the convection zone ( $c f$. Byington et al., 2012)? Does the equatorward tachocline flow in the polar regions and the poleward flow in the equatorial regions advect the periphery of the predominantly dipole remnant field in the radiative interior 
to align with the upwelling flow-convergence zone (Gough, 2012c), or do the torques from the rotational shear dominate the dynamics, as Toby Wood and Michael McIntyre (in preparation) quite plausibly presume? Is such a putative inclined dipole responsible for the active longitudes? - and the emergence of sunspots? Most previous dynamical studies have presumed the tachocline flow to be essentially steadv: the evidence presented recently bv Antia, Chitre, and Gough (2012) suggests that it varies with the solar cycle. So these issues will need to be readdressed in a new light. The rise of sunspots through the convection is now becoming accessible to seismic observation (Ilonidis. Zhao. and Kosovichev, 2011). There are also questions related to structural changes associated with the cycle, and how they are related to the variations in the Sun's outputs. And what of the rotation of the solar core, and the associated circulation, if $\Omega$ differs substantially from the angular velocity of the surrounding envelope? That is likely to influence our ideas about other stars.

It must be appreciated that the Sun is an important benchmark for the whole of stellar physics, and helioseismology for asteroseismology. Finding answers to many of these questions is likely to be assisted by new helioseismological findings, although seismology alone will not be enough. Answers will not come easily the easy questions were answered quickly (although not necessarily easily) in the early days. But the rewards from answering the new questions - designing subtle, hardly detectable, seismic diagnostics and developing techniques to analyse them - are potentially great. Their pursuit is to a large degree the task of the new generation of young seismologists.

\section{Acknowledgements}

I am grateful to Jørgen Christensen-Dalsgaard, Werner Däppen, Günter Houdek, and Sasha Kosovichev for interesting discussion. I thank Jeannette Gilbert and Paula Younger for typing the first draft of this paper, Günter Houdek for providing Figure 4, and Amanda Smith for her help in producing Figures 1 and 6. I thank the Leverhulme Trust for an Emeritus Fellowship, and P.H. Scherrer for support from HMI NASA contract NAS5-02139. This article has benefitted from comments on the first draft by a referee who raised the matter of the reliability of the equation of state.

\section{References}

Abraham, Z., Iben, I. Jr: 1971, More Solar Models and Neutrino Fluxes. Astrophys. J. 170, 157. doi $10.1086 / 151197$

Allende Prieto, C., Lambert, D.L., Asplund, M.: 2001, The Forbidden Abundance of Oxygen in the Sun. Astrophys. J. Lett. 556, L63-L66. doi 10.1086/322874

Allende Prieto, C., Lambert, D.L., Asplund, M.: 2002, A Reappraisal of the Solar Photospheric C/O Ratio. Astrophys. J. Lett. 573, L137-L140. doi 10.1086/342095 
Amelin, Y., Krot, A.N., Hutcheon, I.D., Ulyanov, A.A.: 2002, Lead Isotopic Ages of Chondrules and Calcium-Aluminum-Rich Inclusions. Science 297, 1678-1683. doi:10.1126/science. 1073950

Ando, H., Osaki, Y.: 1975, Nonadiabatic nonradial oscillations - an application to the fiveminute oscillation of the sun. Pub. Astron. Soc. Japan 27, 581-603.

Antia, H.M., Basu, S.: 2004, Temporal Variations in the Solar Radius? In: D. Danesy (ed.) SOHO 14 Helio- and Asteroseismology: Towards a Golden Future, ESA Special Publication 559, 301.

Antia, H.M., Basu, S.: 2006, Determining Solar Abundances Using Helioseismology. Astrophys. J. 644, $1292-1298$. doi $10.1086 / 503707$

Antia, H.M., Basu, S.: 2011, Are recent solar heavy element abundances consistent with helioseismology? Journal of Physics Conference Series 271(1), 012034. doi: $10.1088 / 1742-6596 / 271 / 1 / 012034$

Antia, H.M., Chitre, S.M., Gough, D.O.: 2008, Temporal variations in the Sun's rotational kinetic energy. Astron. Astrophys. 477, 657-663. doi 10.1051/0004-6361:20078209.

Antia, H.M., Chitre, S.M., Gough, D.O.: 2012, On the magnetic field required for driving the observed angular-velocity variations in the solar convection zone. Mon. Not. Roy. Astron. Soc., in press.

Asplund, M.: 2005, New Light on Stellar Abundance Analyses: Departures from LTE and Homogeneity. Ann. Rev. Astron. Astrophys. 43, 481-530. doi:10.1146/annurev.astro.42.053102.134001.

Asplund, M., Grevesse, N., Sauval, A.J.: 2005a, The Solar Chemical Composition. In: T. G. Barnes III \& F. N. Bash (ed.) Cosmic Abundances as Records of Stellar Evolution and Nucleosynthesis, Astronomical Society of the Pacific Conference Series 336, 25.

Asplund, M., Nordlund, A., Trampedach, R., Stein, R.F.: 2000, Line formation in solar granulation. II. The photospheric Fe abundance. Astron. Astrophys. 359, 743-754.

Asplund, M., Grevesse, N., Sauval, A.J., Allende Prieto, C., Kiselman, D.: 2004, Line formation in solar granulation. IV. [O I], O I and $\mathrm{OH}$ lines and the photospheric $\mathrm{O}$ abundance. Astron. Astrophys. 417, $751-768$. doi 10.1051/0004-6361:20034328

Asplund, M., Grevesse, N., Sauval, A.J., Allende Prieto, C., Kiselman, D.: 2005b, Line formation in solar granulation. IV. [O I], O I and $\mathrm{OH}$ lines and the photospheric $\mathrm{O}$ abundance. Astron. Astrophys. 435, 339-340. doi 10.1051/0004-6361:20034328e

Asplund, M., Grevesse, N., Sauval, A.J., Allende Prieto, C., Blomme, R.: 2005c, Line formation in solar granulation. VI. [C I], C I, $\mathrm{CH}$ and $\mathrm{C}_{2}$ lines and the photospheric $\mathrm{C}$ abundance. Astron. Astrophys. 431, 693-705. doi 10.1051/0004-6361:20041951

Asplund, M., Grevesse, N., Sauval, A.J., Scott, P.: 2009, The Chemical Composition of the Sun. Ann. Rev. Astron. Astrophys. 47, 481-522. doi 10.1146/annurev.astro.46.060407.145222

Bahcall, J.N.: 2001, High-energy physics: Neutrinos reveal split personalities. Nature 412 , $29-31$.

Bahcall, J.N., Pinsonneault, M.H.: 1992, Standard solar models, with and without helium diffusion, and the solar neutrino problem. Reviews of Modern Physics 64, 885-926. doi: $10.1103 /$ RevModPhys.64.885

Bahcall, J.N., Ulrich, R.K.: 1971, Solar Neutrinos. III. Composition and Magnetic-Field Effects and Related Inferences. Astrophys. J. 170, 593. doi 10.1086/151245

Bahcall, J.N., Ulrich, R.K.: 1988, Solar models, neutrino experiments, and helioseismology. Reviews of Modern Physics 60, 297 - 372. doi 10.1103/RevModPhys.60.297

Bahcall, J.N., Basu, S., Pinsonneault, M.H.: 1998, How uncertain are solar neutrino predictions? Physics Letters B 433, 1-8. doi 10.1016/S0370-2693(98)00657-1

Bahcall, J.N., Pinsonneault, M.H., Basu, S.: 2001, Solar Models: Current Epoch and Time Dependences, Neutrinos, and Helioseismological Properties. Astrophys. J. 555, 990-1012. doi: $10.1086 / 321493$

Bahcall, J.N., Basu, S., Pinsonneault, M.H., Serenelli, A.M.: 2005, Helioseismological Implications of Recent Solar Abundance Determinations. Astrophys. J. 618, 1049-1056. doi: $10.1086 / 426070$

Balmforth, N.J., Gough, D.O.: 1990, Effluent stellar pulsation. Astrophys. J. 362, 256-266. doi:10.1086/169262

Basu, S.: 1998, Effects of errors in the solar radius on helioseismic inferences. Mon. Not. Roy. Astron. Soc. 298, 719 - 728. doi 10.1046/j.1365-8711.1998.01690.x

Basu, S., Antia, H.M.: 1995, Helium abundance in the solar envelope. Mon. Not. Roy. Astron. Soc. 276, $1402-1408$. 
Basu, S., Antia, H.M.: 1997, Seismic measurement of the depth of the solar convection zone. Mon. Not. Roy. Astron. Soc. 287, 189-198.

Basu, S., Antia, H.M.: 2003, Changes in Solar Dynamics from 1995 to 2002. Astrophys. J. 585, 553-565. doi $10.1086 / 346020$

Basu, S., Antia, H.M.: 2004, Constraining Solar Abundances Using Helioseismology. Astrophys. J. Lett. 606, L85-L88. doi $10.1086 / 421110$

Basu, S., Antia, H.M.: 2008, Helioseismology and solar abundances. Phys. Rep. 457, $217-283$. doi:10.1016/j.physrep.2007.12.002

Basu, S., Mandel, A.: 2004, Does Solar Structure Vary with Solar Magnetic Activity? Astrophys. J. Lett. 617, L155-L158. doi 10.1086/427435

Basu, S., Däppen, W., Nayfonov, A.: 1999, Helioseismic Analysis of the Hydrogen Partition Function in the Solar Interior. Astrophys. J. 518, 985-993. doi 10.1086/307312

Basu, S., Christensen-Dalsgaard, J., Chaplin, W.J., Elsworth, Y., Isaak, G.R., New, R., Schou, J., Thompson, M.J., Tomczyk, S.: 1997, Solar internal sound speed as inferred from combined BiSON and LOWL oscillation frequencies. Mon. Not. Roy. Astron. Soc. 292, 243 .

Basu, S., Chaplin, W.J., Elsworth, Y., New, R., Serenelli, A.M., Verner, G.A.: 2007, Solar Abundances and Helioseismology: Fine-Structure Spacings and Separation Ratios of LowDegree p-Modes. Astrophys. J. 655, 660-671. doi 10.1086/509820

Basu, S., Broomhall, A.M., Chaplin, W.J., Elsworth, Y., Fletcher, S., New, R.: 2010, Differences Between the Current Solar Minimum and Earlier Minima. In: S. R. Cranmer, J. T. Hoeksema, \& J. L. Kohl (ed.) SOHO-23: Understanding a Peculiar Solar Minimum, Astronomical Society of the Pacific Conference Series 428, 37.

Baturin, V.A., Däppen, W., Gough, D.O., Vorontsov, S.V.: 2000, Seismology of the solar envelope: sound-speed gradient in the convection zone and its diagnosis of the equation of state. Mon. Not. Roy. Astron. Soc. 316, $71-83$. doi 10.1046/j.1365-8711.2000.03459.x.

Bonanno, A., Schlattl, H., Paternò, L.: 2002, The age of the Sun and the relativistic corrections in the EOS. Astron. Astrophys. 390, 1115-1118. doi 10.1051/0004-6361:20020749.

Bouvier, A., Wadhwa, M.: 2010, The age of the Solar System redefined by the oldest Pb-Pb age of a meteoritic inclusion. Nature Geoscience 3, $637-641$. doi $10.1038 /$ ngeo941

Brans, C., Dicke, R.H.: 1961, Mach's Principle and a Relativistic Theory of Gravitation. Physical Review 124, 925 - 935. doi 10.1103/PhysRev.124.925

Bretherton, F.P., Spiegel, A.E.: 1968, The Effect of the Convection Zone on Solar Spin-Down. Astrophys. J. Lett. 153, L77. doi $10.1086 / 180224$

Brown, T.M., Christensen-Dalsgaard, J., Dziembowski, W.A., Goode, P., Gough, D.O., Morrow, C.A.: 1989, Inferring the sun's internal angular velocity from observed p-mode frequency splittings. Astrophys. J. 343, 526-546. doi 10.1086/167727.

Byington, B., Stone, J., Brummell, N., Gough, D.O.: 2012, Stoked nondynamos: sustaining field in magnetically non-closed systems . Solar Physics. this issue.

Caffau, E., Maiorca, E., Bonifacio, P., Faraggiana, R., Steffen, M., Ludwig, H.G., Kamp, I., Busso, M.: 2009, The solar photospheric nitrogen abundance. Analysis of atomic transitions with 3D and 1D model atmospheres. Astron. Astrophys. 498, 877-884. doi:10.1051/0004-6361/200810859

Caffau, E., Ludwig, H.G., Steffen, M., Freytag, B., Bonifacio, P.: 2011, Solar Chemical Abundances Determined with a CO5BOLD 3D Model Atmosphere. Solar Phys. 268, 255-269. doi: $10.1007 /$ s11207-010-9541-4.

Chaplin, W.J., Elsworth, Y., Howe, R., Isaak, G.R., McLeod, C.P., Miller, B.A., van der Raay, H.B., Wheeler, S.J., New, R.: 1996, BiSON Performance. Solar Phys. 168, 1-18. doi:10.1007/BF00145821.

Chaplin, W.J., Christensen-Dalsgaard, J., Elsworth, Y., Howe, R., Isaak, G.R., Larsen, R.M., New, R., Schou, J., Thompson, M.J., Tomczyk, S.: 1999, Rotation of the solar core from BiSON and LOWL frequency observations. Mon. Not. Roy. Astron. Soc. 308, 405-414. doi: 10.1046/j.1365-8711.1999.02691.x.

Chaplin, W.J., Serenelli, A.M., Basu, S., Elsworth, Y., New, R., Verner, G.A.: 2007, Solar Heavy-Element Abundance: Constraints from Frequency Separation Ratios of Low-Degree p-Modes. Astrophys. J. 670, $872-884$. doi $10.1086 / 522578$

Christensen-Dalsgaard, J.: 1992, Solar models with enhanced energy transport in the core. Astrophys. J. 385, 354-362. doi 10.1086/170944

Christensen-Dalsgaard, J., Gough, D.O.: 1976, Towards a heliological inverse problem. Nature 259, 89-92. doi $10.1038 / 259089 a 0$ 
Christensen-Dalsgaard, J., Gough, D.O.: 1980, Implications of the whole-disk Doppler observations of the sun. In: Hill, H.A., Dziembowski, W.A. (eds.) Nonradial and Nonlinear Stellar Pulsation, Lecture Notes in Physics, Berlin Springer Verlag 125, 184-190. doi:10.1007/3-540-09994-8_18

Christensen-Dalsgaard, J., Gough, D.O.: 1981, Comparison of observed solar whole-disk oscillation frequencies with the predictions of a sequence of solar models. Astron. Astrophys. 104, $173-176$

Christensen-Dalsgaard, J., Houdek, G.: 2010, Prospects for asteroseismology. Astrophys. Space Sci. 328, 51 -66. doi $10.1007 /$ s10509-009-0227-z

Christensen-Dalsgaard, J., Gough, D.O., Morgan, J.G.: 1979a, Dirty solar models. Astron. Astrophys. 73, $121-128$.

Christensen-Dalsgaard, J., Gough, D.O., Morgan, J.G.: 1979b, Erratum: "Dirty solar models" [Astron. Astrophys., Vol. 79, p. 121 - 128 (1979)]. Astron. Astrophys. 79, 260.

Christensen-Dalsgaard, J., Gough, D.O., Thompson, M.J.: 1989, Differential asymptotic soundspeed inversions. Mon. Not. Roy. Astron. Soc. 238, 481-502.

Christensen-Dalsgaard, J., Gough, D.O., Thompson, M.J.: 1991, The depth of the solar convection zone. Astrophys. J. 378, 413-437. doi:10.1086/170441

Christensen-Dalsgaard, J., Proffitt, C.R., Thompson, M.J.: 1993, Effects of diffusion on solar models and their oscillation frequencies. Astrophys. J. Lett. 403, L75-L78. doi: $10.1086 / 186725$

Christensen-Dalsgaard, J., Duvall, T.L. Jr, Gough, D.O., Harvey, J.W., Rhodes, E.J. Jr: 1985, Speed of sound in the solar interior. Nature 315, 378-382. doi $10.1038 / 315378 a 0$

Christensen-Dalsgaard, J., Däppen, W., Ajukov, S.V., Anderson, E.R., Antia, H.M., Basu, S., Baturin, V.A., Berthomieu, G., Chaboyer, B., Chitre, S.M., Cox, A.N., Demarque, P., Donatowicz, J., Dziembowski, W.A., Gabriel, M., Gough, D.O., Guenther, D.B., Guzik, J.A., Harvey, J.W., Hill, F., Houdek, G., Iglesias, C.A., Kosovichev, A.G., Leibacher, J.W., Morel, P., Proffitt, C.R., Provost, J., Reiter, J., Rhodes, E.J. Jr, Rogers, F.J., Roxburgh, I.W., Thompson, M.J., Ulrich, R.K.: 1996, The Current State of Solar Modeling. Science 272, 1286 - 1292. doi 10.1126 /science. 272.5266 .1286

Christensen-Dalsgaard, J., Di Mauro, M.P., Houdek, G., Pijpers, F.: 2009, On the opacity change required to compensate for the revised solar composition. Astron. Astrophys. 494, 205 - 208. doi 10.1051/0004-6361:200810170

Christensen-Dalsgaard, J., Monteiro, M.J.P.F.G., Rempel, M., Thompson, M.J.: 2011, A more realistic representation of overshoot at the base of the solar convective envelope as seen by helioseismology. Mon. Not. Roy. Astron. Soc. 414, 1158-1174. doi:10.1111/j.1365-2966.2011.18460.x

Claverie, A., Isaak, G.R., McLeod, C.P., van der Raay, H.B., Roca-Cortes, T.: 1980, The Latest Results of the Velocity Spectroscopy of the Sun. In: H. A. Hill \& W. A. Dziembowski (ed.) Nonradial and Nonlinear Stellar Pulsation, Lecture Notes in Physics, Berlin Springer Verlag 125, 181. doi 10.1007/3-540-09994-8_17.

Cox, A.N., Morgan, S.M., Rogers, F.J., Iglesias, C.A.: 1992, An opacity mechanism for the pulsations of OB stars. Astrophys. J. 393, 272-277. doi $10.1086 / 171504$

Däppen, W.: 1998, Microphysics: Equation of State. Space Sci. Rev. 85, 49-60. doi: $10.1023 / \mathrm{A}: 1005176317912$

Däppen, W.: 2004, Equations of state for solar and stellar modeling. In: V. Celebonovic, D. Gough, \& W. Däppen (ed.) Equation-of-State and Phase-Transition in Models of Ordinary Astrophysical Matter, American Institute of Physics Conference Series 731, 3-17. doi: $10.1063 / 1.1828391$

Däppen, W.: 2007, Seismic Abundance Determination in the Sun and in Stars. In: R. J. Stancliffe, G. Houdek, R. G. Martin, \& C. A. Tout (ed.) Unsolved Problems in Stellar Physics: A Conference in Honour of Douglas Gough, American Institute of Physics Conference Series 948, 179 - 190. doi $10.1063 / 1.2818968$

Däppen, W., Gough, D.O.: 1984, On the Determination of the Helium Abundance of the Solar Convection Zone. In: Liege International Astrophysical Colloquia, Liege International Astrophysical Colloquia 25, 264-268.

Däppen, W., Gilliland, R.L., Christensen-Dalsgaard, J.: 1986, Weakly interacting massive particles, solar neutrinos, and solar oscillations. Nature 321, 229-231. doi 10.1038/321229a0

Däppen, W., Gough, D.O., Thompson, M.J.: 1988, Further progress on the helium abundance determination. In: Rolfe, E.J. (ed.) Seismology of the Sun and Sun-Like Stars, ESA Special Publication 286, 505-510. 
Däppen, W., Gough, D.O., Kosovichev, A.G., Thompson, M.J.: 1991, A New Inversion for the Hydrostatic Stratification of the Sun. In: Gough, D., Toomre, J. (eds.) Challenges to Theories of the Structure of Moderate-Mass Stars, Lecture Notes in Physics, Berlin Springer Verlag 388, 111. doi 10.1007/3-540-54420-8_57.

Deubner, F.L.: 1975, Observations of low wavenumber nonradial eigenmodes of the sun. Astron. Astrophys. 44, 371-375.

Di Mauro, M.P., Christensen-Dalsgaard, J., Rabello-Soares, M.C., Basu, S.: 2002, Inferences on the solar envelope with high-degree modes. Astron. Astrophys. 384, 666-677. doi: 10.1051/0004-6361:20020020

Dicke, R.H.: 1967, The Solar Spin-Down Problem. Astrophys. J. Lett. 149, L121. doi:10.1086/180072

Dicke, R.H.: 1970, The Solar Oblateness and the Gravitational Quadrupole Moment. Astrophys. J. 159, 1. doi $10.1086 / 150286$.

Dicke, R.H., Goldenberg, H.M.: 1967, Solar Oblateness and General Relativity. Physical Review Letters 18, 313-316. doi 10.1103/PhysRevLett.18.313

Dicke, R.H., Goldenberg, H.M.: 1974, The Oblateness of the Sun. Astrophys. J. Suppl. 27, 131. doi $10.1086 / 190292$

Doğan, G., Bonanno, A., Christensen-Dalsgaard, J.: 2010, Near-surface effects and solar-age determination. ArXiv e-prints.

Drake, J.J., Testa, P.: 2005, The 'solar model problem' solved by the abundance of neon in nearby stars. Nature 436, 525-528. doi $10.1038 /$ nature03803

Duvall, T.L. Jr, Harvey, J.W.: 1983, Observations of solar oscillations of low and intermediate degree. Nature 302, 24-27. doi:10.1038/302024a0

Duvall, T.L. Jr, Dziembowski, W.A., Goode, P.R., Gough, D.O., Harvey, J.W., Leibacher, J.W.: 1984, Internal rotation of the sun. Nature 310, 22-25. doi 10.1038/310022a0

Dziembowski, W.A., Goode, P.R.: 2004, Helioseismic Probing of Solar Variability: The Formalism and Simple Assessments. Astrophys. J. 600, 464-479. doi $10.1086 / 379708$

Dziembowski, W.A., Goode, P.R.: 2005, Sources of Oscillation Frequency Increase with Rising Solar Activity. Astrophys. J. 625, 548-555. doi $10.1086 / 429712$

Dziembowski, W.A., Pamyatnykh, A.A.: 1993, The opacity mechanism in B-type stars. I Unstable modes in Beta Cephei star models. Mon. Not. Roy. Astron. Soc. 262, $204-212$.

Dziembowski, W.A., Moskalik, P., Pamyatnykh, A.A.: 1993, The Opacity Mechanism in BType Stars - Part Two - Excitation of High-Order G-Modes in Main Sequence Stars. Mon. Not. Roy. Astron. Soc. 265, 588.

Dziembowski, W.A., Moskalik, P., Pamyatnykh, A.A.: 1994, G-Mode instability in the main sequence B-type stars. In: L. A. Balona, H. F. Henrichs, \& J. M. Le Contel (ed.) Pulsation; Rotation; and Mass Loss in Early-Type Stars, IAU Symposium 162, 69.

Dziembowski, W.A., Pamyatnykh, A.A., Sienkiewicz, R.: 1990, Solar model from helioseismology and the neutrino flux problem. Mon. Not. Roy. Astron. Soc. 244, 542-550.

Dziembowski, W.A., Fiorentini, G., Ricci, B., Sienkiewicz, R.: 1999, Helioseismology and the solar age. Astron. Astrophys. 343, $990-996$.

Elliott, J.R.: 1995, Opacity Determination in the Solar Radiative Interior. Mon. Not. Roy. Astron. Soc. 277, 1567.

Elliott, J.R., Gough, D.O., Sekii, T.: 1998, Helioseismic Determination of the Solar Tachocline Thickness. In: S. Korzennik (ed.) Structure and Dynamics of the Interior of the Sun and Sun-like Stars, ESA Special Publication 418, 763.

Elsworth, Y., Howe, R., Isaak, G.R., McLeod, C.P., Miller, B.A., New, R., Wheeler, S.J., Gough, D.O.: 1995, Slow rotation of the Sun's interior. Nature 376, 669-672. doi: $10.1038 / 376669 a 0$

Emilio, M., Bush, R.I., Kuhn, J., Scherrer, P.: 2007, A Changing Solar Shape. Astrophys. J. Lett. 660, L161-L163. doi $10.1086 / 518212$.

Faulkner, J., Gough, D.O., Vahia, M.N.: 1986, Weakly interacting massive particles and solar oscillations. Nature 321, 226-229. doi 10.1038/321226a0

Fivian, M.D., Hudson, H.S., Lin, R.P., Zahid, H.J.: 2008, A Large Excess in Apparent Solar Oblateness Due to Surface Magnetism. Science 322, 560. doi 10.1126/science.1160863

Fossat, E., Grec, G., Pomerantz, M.: 1981, Solar pulsations observed from the geographic South Pole - Initial results. Solar Phys. 74, 59-63. doi:10.1007/BF00151274.

Foukal, P., Fröhlich, C., Spruit, H., Wigley, T.M.L.: 2006, Variations in solar luminosity and their effect on the Earth's climate. Nature 443, 161-166. doi 10.1038 /nature05072

Fröhlich, C.: 2011, Total Solar Irradiance: What Have We Learned from the Last Three Cycles and the Recent Minimum? Space Sci. Rev., 133. doi 10.1007/s11214-011-9780-1 
Gilliland, R.L., Faulkner, J., Press, W.H., Spergel, D.N.: 1986, Solar models with energy transport by weakly interacting particles. Astrophys. J. 306, 703-709. doi 10.1086/164380

Gizon, L., Schunker, H., Baldner, C.S., Basu, S., Birch, A.C., Bogart, R.S., Braun, D.C., Cameron, R., Duvall, T.L., Hanasoge, S.M., Jackiewicz, J., Roth, M., Stahn, T., Thompson, M.J., Zharkov, S.: 2009, Helioseismology of Sunspots: A Case Study of NOAA Region 9787. Space Sci. Rev. 144, 249-273. doi 10.1007/s11214-008-9466-5

Goode, P.R., Didkovsky, L.V., Libbrecht, K.G., Woodard, M.F.: 2002, Evolution of the sun's near-surface asphericities over the activity cycle. Advances in Space Research 29, 18891898. doi 10.1016/S0273-1177(02)00240-5

Gough, D.O.: 1977, Random remarks on solar hydrodynamics. In: B. Bonnet \& P. Delache (ed.) IAU Colloq. 36: The Energy Balance and Hydrodynamics of the Solar Chromosphere and Corona, G. de Bussac, Clermont-Ferrand, 3-36.

Gough, D.O.: 1978, The relevance of solar oscillations to theories of the rotation of the Sun. In: G. Belvedere \& L. Paternò (ed.) Proc. EPS Workshop on Solar Rotation, Catania University Press, $87-103$.

Gough, D.O.: 1981, A new measure of the solar rotation. Mon. Not. Roy. Astron. Soc. 196, $731-745$.

Gough, D.O.: 1982a, A Review of the Theory of Solar Oscillations and its Implications concerning the internal Structure of the Sun. In: Cox, J.P., Hansen, C.J. (eds.) Pulsations in Classical and Cataclysmic Variable Stars, 117.

Gough, D.O.: 1982b, Inferences from solar oscillations. Irish A.J. 15, 118-119.

Gough, D.O.: 1983a, Our First Inferences from Helioseismology. Physics Bulletin 34, 502-507.

Gough, D.O.: 1983b, Solar structure: A bridge in a gap in solar oscillations. Nature 302, 18. doi $10.1038 / 302018 \mathrm{a} 0$

Gough, D.O.: 1983c, The protosolar helium abundance. In: P. A. Shaver, D. Kunth, \& K. Kjar (ed.) Primordial Helium, 117-136.

Gough, D.O.: 1984a, Helioseismology. The Observatory 104, 118-119.

Gough, D.O.: 1984b, Towards a solar model. Mem. Soc. Astron. Ital. 55, 13.

Gough, D.O.: 1986, EBK Quantization of Stellar Waves. In: Y. Osaki (ed.) Hydrodynamic and Magnetodynamic Problems in the Sun and Stars, Univ. Tokyo Press, 117-143.

Gough, D.O.: 1990, The internal structure of late-type main-sequence stars. In: B. Gustafsson \& P. E. Nissen (ed.) Astrophysics: Recent Progress and Future Possibilities (A91-15054 03-90), Kongelige Danske Videnskabernes Selskab, Mat-fys Medd, 42:4, 13-50.

Gough, D.O.: 1995, Prospects for Asteroseismic Inference. In: R. K. Ulrich, E. J. Rhodes Jr, \& W. Däppen (ed.) GONG 1994: Helio- and Astro-Seismology from the Earth and Space, Astronomical Society of the Pacific Conference Series 76, 551.

Gough, D.O.: 2004, The power of helioseismology to address issues of fundamental physics. In: V. Celebonović, D. Gough, \& W. Däppen (ed.) Equation-of-State and Phase-Transition in Models of Ordinary Astrophysical Matter, American Institute of Physics Conference Series 731, $119-138$. doi $10.1063 / 1.1828398$

Gough, D.O.: 2006, Helioseismological Determination of the State of the Solar Interior. In: H. Lacoste \& L. Ouwehand (ed.) SOHO-17: 10 Years of SOHO and Beyond, ESA SP-617 $\mathbf{1}, 1-17$.

Gough, D.O.: 2012a, Heliophysics gleaned from seismology. In: H. Shibahashi \& M. Takata (ed.) Progress in solar/stellar Physics with Helio- and Asteroseismology; Proc. 65th Fujihara Seminar, Astron. Soc. Pacific Conf. Ser. in press.

Gough, D.O.: 2012b, How oblate is the Sun? Science 337, 1611-1612. doi:10.1126/science. 1226988

Gough, D.O.: 2012c, Pattern formation in rapidly oscillating peculiar A stars. Geophysical and Astrophysical Fluid Dynamics 106, 429-449.

Gough, D.O., Hindman, B.W.: 2010, Helioseismic Detection of Deep Meridional Flow. Astrophys. J. 714, $960-970$. doi 10.1088/0004-637X/714/1/960

Gough, D.O., Kosovichev, A.G.: 1988, An attempt to understand the Stanford p-mode data. In: Rolfe, E.J. (ed.) Seismology of the Sun and Sun-Like Stars, ESA Special Publication 286, $195-201$.

Gough, D.O., Kosovichev, A.G.: 1990, Using Helioseismic Data to Probe the Hydrogen Abundance in the Solar Core. In: Berthomieu, G., Cribier, M. (eds.) IAU Colloq. 121: Inside the Sun, Astrophysics and Space Science Library 159, 327.

Gough, D.O., McIntyre, M.E.: 1998, Inevitability of a magnetic field in the Sun's radiative interior. Nature 394, $755-757$. doi $10.1038 / 29472$ 
Gough, D.O., Scherrer, P.H.: 2002, In: Bleeker, J.A., Geiss, J., Huber, M.C.E. (eds.) The solar interior, The Century of Space Science, Volume I, Kluwer Academic Publishers, Dordrecht, ???, 1035.

Gough, D.O., Sekii, T., Stark, P.B.: 1996a, Inferring Spatial Variation of Solar Properties from Helioseismic Data. Astrophys. J. 459, 779. doi $10.1086 / 176942$

Gough, D.O., Kosovichev, A.G., Toomre, J., Anderson, E., Antia, H.M., Basu, S., Chaboyer, B., Chitre, S.M., Christensen-Dalsgaard, J., Dziembowski, W.A., Eff-Darwich, A., Elliott, J.R., Giles, P.M., Goode, P.R., Guzik, J.A., Harvey, J.W., Hill, F., Leibacher, J.W., Monteiro, M.J.P.F.G., Richard, O., Sekii, T., Shibahashi, H., Takata, M., Thompson, M.J., Vauclair, S., Vorontsov, S.V.: 1996b, The Seismic Structure of the Sun. Science 272, 1296-1300. doi $10.1126 /$ science. 272.5266 .1296

Grec, G., Fossat, E., Pomerantz, M.: 1980, Solar oscillations - Full disk observations from the geographic South Pole. Nature 288, 541-544. doi $10.1038 / 288541 a 0$.

Grevesse, N., Noels, A.: 1993, Cosmic abundances of the elements. In: N. Prantzos, E. VangioniFlam, \& M. Casse (ed.) Origin and Evolution of the Elements, 15-25.

Grevesse, N., Sauval, A.J.: 1998, Standard Solar Composition. Space Sci. Rev. 85, 161-174. doi: $10.1023 / \mathrm{A}: 1005161325181$

Grevesse, N., Asplund, M., Sauval, A.J., Scott, P.: 2011, The New Solar Composition and the Solar Metallicity. In: Miralles, M. P. \& Sánchez Almeida, J. (ed.) The Sun, the Solar Wind, and the Heliosphere, 51.

Guzik, J.A., Watson, L.S., Cox, A.N.: 2005, Can Enhanced Diffusion Improve Helioseismic Agreement for Solar Models with Revised Abundances? Astrophys. J. 627, 1049-1056. doi $10.1086 / 430438$

Guzik, J.A., Watson, L.S., Cox, A.N.: 2006, Implications of revised solar abundances for helioseismology. Mem. Soc. Astron. Ital. 77, 389.

Hampel, W., Heusser, G., Kiko, J., Kirsten, T., Laubenstein, M., Pernicka, E., Rau, W., Rönn, U., Schlosser, C., Wojcik, M., Zakharov, Y., v. Ammon, R., Ebert, K.H., Fritsch, T., Heidt, D., Henrich, E., Stieglitz, L., Weirich, F., Balata, M., Sann, M., Hartmann, F.X., Bellotti, E., Cattadori, C., Cremonesi, O., Ferrari, N., Fiorini, E., Zanotti, L., Altmann, M., v. Feilitzsch, F., Mößbauer, R., Berthomieu, G., Schatzman, E., Carmi, I., Dostrovsky, I., Bacci, C., Belli, P., Bernabei, R., D’Angelo, S., Paoluzi, L., Bevilacqua, A., Cribier, M., Gosset, L., Rich, J., Spiro, M., Tao, C., Vignaud, D., Boger, J., Hahn, R.L., Rowley, J.K., Stoenner, R.W., Weneser, J.: 1996, GALLEX solar neutrino observations: Results for GALLEX III. Physics Letters B 388, 384-396. doi:10.1016/S0370-2693(96)01121-5.

Hindman, B.W., Haber, D.A., Toomre, J.: 2009, Subsurface Circulations within Active Regions. Astrophys. J. 698, 1749-1760. doi 10.1088/0004-637X/698/2/1749

Houdek, G., Gough, D.O.: 2007, An asteroseismic signature of helium ionization. Mon. Not. Roy. Astron. Soc. 375, 861-880. doi 10.1111/j.1365-2966.2006.11325.x

Houdek, G., Gough, D.O.: 2011, On the seismic age and heavy-element abundance of the Sun. Mon. Not. Roy. Astron. Soc. 418, $1217-1230$. doi 10.1111/j.1365-2966.2011.19572.x

Howard, L.N., Moore, D.W., Spiegel, E.A.: 1967, . Nature 214, 1297.

Howe, R., Thompson, M.J.: 1996, On the use of the error correlation function in helioseismic inversions. Mon. Not. Roy. Astron. Soc. 281, 1385.

Howe, R., Komm, R., Hill, F., Ulrich, R., Haber, D.A., Hindman, B.W., Schou, J., Thompson, M.J.: 2006a, Large-Scale Zonal Flows Near the Solar Surface. Solar Phys. 235, 1-15. doi: $10.1007 /$ s11207-006-0117-2.

Howe, R., Rempel, M., Christensen-Dalsgaard, J., Hill, F., Komm, R., Larsen, R.M., Schou, J., Thompson, M.J.: 2006b, Solar Convection Zone Dynamics: How Sensitive Are Inversions to Subtle Dynamo Features? Astrophys. J. 649, 1155-1168. doi 10.1086/506931.

Iglesias, C.A., Rogers, F.J.: 1991, Opacities for the solar radiative interior. Astrophys. J. 371, 408-417. doi $10.1086 / 169902$

Iglesias, C.A., Rogers, F.J.: 1996, Updated Opal Opacities. Astrophys. J. 464, 943. doi $10.1086 / 177381$

Iglesias, C.A., Rogers, F.J., Wilson, B.G.: 1990, Opacities for classical Cepheid models. Astrophys. J. 360, 221-226. doi 10.1086/169110

Ilonidis, S., Zhao, J., Kosovichev, A.: 2011, Detection of Emerging Sunspot Regions in the Solar Interior. Science 333, 993. doi 10.1126/science.1206253

Jacobsen, B., Yin, Q.Z., Moynier, F., Amelin, Y., Krot, A.N., Nagashima, K., Hutcheon, I.D., Palme, H.: 2008, ${ }^{26} \mathrm{Al}{ }^{26} \mathrm{Mg}$ and ${ }^{207} \mathrm{~Pb}{ }^{206} \mathrm{~Pb}$ systematics of Allende CAIs: Canonical solar initial ${ }^{26} \mathrm{Al} /{ }^{27} \mathrm{Al}$ ratio reinstated. Earth and Planetary Science Letters 272, 353-364. doi:10.1016/j.epsl.2008.05.003 
Jacobsen, B., Yin, Q.Z., Moynier, F., Amelin, Y., Krot, A.N., Nagashima, K., Hutcheon, I.D., Palme, H.: 2009, Erratum to ${ }^{26} \mathrm{Al}{ }^{26} \mathrm{Mg}$ and ${ }^{207} \mathrm{~Pb}{ }^{206} \mathrm{~Pb}$ systematics of Allende CAIs: Canonical solar initial ${ }^{26} \mathrm{Al} /{ }^{27} \mathrm{Al}$ ratio reinstated" [Earth Planet Sci. Lett. 272 (2008) 353 364]. Earth and Planetary Science Letters 277, 549-549. doi 10.1016/j.epsl.2008.12.001

Kiriakidis, M., El Eid, M.F., Glatzel, W.: 1992, Heavy element opacities and the pulsations of Beta Cepheid stars. Mon. Not. Roy. Astron. Soc. 255, 1P-5P.

Kitiashvili, I.N., Kosovichev, A.G., Wray, A.A., Mansour, N.N.: 2009, Traveling Waves of Magnetoconvection and the Origin of the Evershed Effect in Sunspots. Astrophys. J. Lett. 700, L178 - L181. doi 10.1088/0004-637X/700/2/L178

Kitiashvili, I.N., Bellot Rubio, L.R., Kosovichev, A.G., Mansour, N.N., Sainz Dalda, A., Wray, A.A.: 2010, Explanation of the Sea-serpent Magnetic Structure of Sunspot Penumbrae. Astrophys. J. Lett. 716, L181-L184. doi 10.1088/2041-8205/716/2/L181

Komm, R., Howe, R., Hill, F.: 2006, Helioseismic sensing of the solar cycle. Advances in Space Research 38, $845-855$. doi $10.1016 /$ j.asr.2005.07.034

Kopp, G., Lean, J.L.: 2011, A new, lower value of total solar irradiance: Evidence and climate significance. Geophys. Res. Lett. 38, 1706. doi 10.1029/2010GL045777

Korzennik, S.G., Ulrich, R.K.: 1989, Seismic analysis of the solar interior. I - Can opacity changes improve the theoretical frequencies? Astrophys. J. 339, 1144-1155. doi $10.1086 / 167369$

Kosovichev, A.G.: 2009, Photospheric and Subphotospheric Dynamics of Emerging Magnetic Flux. Space Sci. Rev. 144, 175-195. doi 10.1007/s11214-009-9487-8

Kosovichev, A.G., Duvall, T.L. Jr: 2011, Investigation of a Sunspot Complex by Helioseismology. ArXiv e-prints.

Kosovichev, A.G., Christensen-Dalsgaard, J., Däppen, W., Dziembowski, W.A., Gough, D.O., Thompson, M.J.: 1992, Sources of uncertainty in direct seismological measurements of the solar helium abundance. Mon. Not. Roy. Astron. Soc. 259, 536-558.

Kuhn, J., Bush, R., Emilio, M., Scholl, I.: 2012, The precise solar shape and its variability. Science 337, 1638-1640. doi $10.1126 /$ science.1223231

Lefebvre, S., Kosovichev, A.G.: 2005, Changes in the Subsurface Stratification of the Sun with the 11-Year Activity Cycle. Astrophys. J. Lett. 633, L149-L152. doi 10.1086/498305

Lefebvre, S., Kosovichev, A.G., Rozelot, J.P.: 2007, Helioseismic Test of Nonhomologous Solar Radius Changes with the 11 Year Activity Cycle. Astrophys. J. Lett. 658, L135-L138. doi: $10.1086 / 515394$

Lodders, K., Palme, H., Gail, H.P.: 2009, Abundances of the Elements in the Solar System. In: J. E. Trümper (ed.) "Landolt-Börnstein - Group VI Astronomy and Astrophysics Numerical Data and Functional Relationships in Science and Technology Volume, 44. doi:10.1007/978-3-540-88055-4_34

Lopes, I.P., Gough, D.O.: 2001, Seismology of stellar envelopes: probing the outer layers of a star through the scattering of acoustic waves. Mon. Not. Roy. Astron. Soc. 322, 473-485. doi $10.1046 /$ j.1365-8711.2001.03940.x.

Moskalik, P., Dziembowski, W.A.: 1992, New opacities and the origin of the Beta Cephei pulsation. Astron. Astrophys. 256, L5-L8.

Moskalik, P., Buchler, J.R., Marom, A.: 1992, Toward a resolution of the bump and beat Cepheid mass discrepancies. Astrophys. J. 385, 685-693. doi 10.1086/170975

Mussack, K., Däppen, W.: 2010, Dynamic screening in solar and stellar nuclear reactions. Astrophys. Space Sci. 328, 153-156. doi 10.1007/s10509-009-0245-x

Mussack, K., Däppen, W.: 2011, Dynamic Screening Correction for Solar p-p Reaction Rates. Astrophys. J. 729, 96. doi 10.1088/0004-637X/729/2/96

Mussack, K., Gough, D.O.: 2009, Measuring Solar Abundances with Seismology. In: M. Dikpati, T. Arentoft, I. González Hernández, C. Lindsey, \& F. Hill (ed.) Solar-Stellar Dynamos as Revealed by Helio- and Asteroseismology: GONG 2008/SOHO 21, Astronomical Society of the Pacific Conference Series 416, 203.

Pijpers, F.P.: 1998, Helioseismic determination of the solar gravitational quadrupole moment. Mon. Not. Roy. Astron. Soc. 297, L76-L80. doi 10.1046/j.1365-8711.1998.01801.x

Ramsey, A.: 1937, Dynamics Part II: Cambridge Univ. Press.

Richard, O., Dziembowski, W.A., Sienkiewicz, R., Goode, P.R.: 1998, Precise Determination of the Solar Helium Abundance by Helioseismology. In: S. Korzennik (ed.) Structure and Dynamics of the Interior of the Sun and Sun-like Stars, ESA Special Publication 418, 517.

Rogers, F.J., Nayfonov, A.: 2002, Updated and Expanded OPAL Equation-of-State Tables: Implications for Helioseismology. Astrophys. J. 576, 1064-1074. doi 10.1086/341894 
Saio, H.: 1992, Opacity in the solar radiative interior inferred from 5-min oscillations. Mon. Not. Roy. Astron. Soc. 258, $491-496$.

Salpeter, E.E.: 1954, Electron screening and thermonuclear reactions. Australian Journal of Physics 7, 373 .

Schou, J., Woodard, M.F., Birch, A.C.: 2009, Large-Scale Flows from Eigenfunction Fitting. In: AAS/Solar Physics Division Meeting, AAS/Solar Physics Division Meeting 40, $07-05$.

Schou, J., Antia, H.M., Basu, S., Bogart, R.S., Bush, R.I., Chitre, S.M., Christensen-Dalsgaard, J., di Mauro, M.P., Dziembowski, W.A., Eff-Darwich, A., Gough, D.O., Haber, D.A., Hoeksema, J.T., Howe, R., Korzennik, S.G., Kosovichev, A.G., Larsen, R.M., Pijpers, F.P., Scherrer, P.H., Sekii, T., Tarbell, T.D., Title, A.M., Thompson, M.J., Toomre, J.: 1998, Helioseismic Studies of Differential Rotation in the Solar Envelope by the Solar Oscillations Investigation Using the Michelson Doppler Imager. Astrophys. J. 505, 390-417. doi:10.1086/306146

Simon, N.R.: 1982, A plea for reexamining heavy element opacities in stars. Astrophys. J. Lett. 260, L87-L90. doi $10.1086 / 183876$

Spergel, D.N., Press, W.H.: 1985, Effect of hypothetical, weakly interacting, massive particles on energy transport in the solar interior. Astrophys. J. 294, 663-673. doi 10.1086/163336.

Spiegel, E.A., Zahn, J.P.: 1992, The solar tachocline. Astron. Astrophys. 265, 106-114.

Steigman, G.: 2007, Primordial Nucleosynthesis in the Precision Cosmology Era. Annual Review of Nuclear and Particle Science 57, 463-491. doi:10.1146/annurev.nucl.56.080805.140437.

Stein, R.F., Nordlund, A.: 1998, Simulations of Solar Granulation. I. General Properties. Astrophys. J. 499, 914. doi $10.1086 / 305678$

Takata, M., Gough, D.O.: 2001, The influence of uncertainties in the Sun's radius on inversions for the solar structure. In: Wilson, A., Pallé, P.L. (eds.) SOHO 10/GONG 2000 Workshop: Helio- and Asteroseismology at the Dawn of the Millennium, ESA Special Publication 464, $543-546$.

Takata, M., Gough, D.O.: 2003, The seismic radius of the Sun, and structure inversions. In: Sawaya-Lacoste, H. (ed.) GONG+ 2002. Local and Global Helioseismology: the Present and Future, ESA Special Publication 517, 397-400.

Tassoul, M.: 1980, Asymptotic approximations for stellar nonradial pulsations. Astrophys. J. Suppl. 43, 469-490. doi $10.1086 / 190678$

Taylor, S.F., Varsik, J.R., Woodard, M.F., Libbrecht, K.G.: 1998, Spatial Dependence of SolarCycle Changes in the Sun's Luminosity. Solar Phys. 178, 1-12.

Tomczyk, S., Schou, J., Thompson, M.J.: 1995a, Measurement of the Rotation Rate in the Deep Solar Interior. Astrophys. J. Lett. 448, L57. doi 10.1086/309598

Tomczyk, S., Streander, K., Card, G., Elmore, D., Hull, H., Cacciani, A.: 1995b, An Instrument to Observe Low-Degree Solar Oscillations. Solar Phys. 159, 1-21. doi: $10.1007 /$ BF00733027.

Tripathy, S.C., Christensen-Dalsgaard, J.: 1998, Opacity effects on the solar interior. I. Solar structure. Astron. Astrophys. 337, 579-590.

Tripathy, S.C., Basu, S., Christensen-Dalsgaard, J.: 1998, Helioseismic determination of opacity corrections. In: J. Provost \& F-X. Schmider (ed.) Sounding solar and stellar interiors: IAU Symposium 181, Poster volume, Observatoire de la Côte d'Azur, Université de Nice, $129-130$.

Turck-Chièze, S., Däppen, W., Fossat, E., Provost, J., Schatzman, E., Vignaud, D.: 1993, The solar interior. Physics Reports 230, 57-235. doi 10.1016/0370-1573(93)90020-E

Ulrich, R.K., Rhodes, E.J. Jr: 1977, The sensitivity of nonradial P mode eigenfrequencies to solar envelope structure. Astrophys. J. 218, 521 -529. doi $10.1086 / 155705$

Vandakurov, Y.V.: 1967, The Frequency Distribution of Stellar Oscillations. Astron. Zh. 44, 786.

Verner, G.A., Chaplin, W.J., Elsworth, Y.: 2006, BiSON Data Show Change in Solar Structure with Magnetic Activity. Astrophys. J. Lett. 640, L95-L98. doi 10.1086/503101

Vorontsov, S.V., Baturin, V.A., Pamyatnykh, A.A.: 1992, Seismology of the solar envelope Towards the calibration of the equation of state. Mon. Not. Roy. Astron. Soc. 257, 32-46.

Vorontsov, S.V., Christensen-Dalsgaard, J., Schou, J., Strakhov, V.N., Thompson, M.J.: 2002, Helioseismic Measurement of Solar Torsional Oscillations. Science 296, 101-103. doi $10.1126 /$ science. 1069190

Weiss, N.O., Thomas, J.H., Brummell, N.H., Tobias, S.M.: 2004, The Origin of Penumbral Structure in Sunspots: Downward Pumping of Magnetic Flux. Astrophys. J. 600, 10731090. doi $10.1086 / 380091$ 
Willson, R.C., Hudson, H.S.: 1988, Solar luminosity variations in solar cycle 21. Nature 332, 810 - 812. doi $10.1038 / 332810 \mathrm{a} 0$

Zhao, J., Kosovichev, A.G.: 2003, Helioseismic Observation of the Structure and Dynamics of a Rotating Sunspot Beneath the Solar Surface. Astrophys. J. 591, 446-453. doi $10.1086 / 375343$

Zhao, J., Kosovichev, A.G., Duvall, T.L. Jr: 2001, Investigation of Mass Flows beneath a Sunspot by Time-Distance Helioseismology. Astrophys. J. 557, 384-388. doi $10.1086 / 321491$

Zhao, J., Kosovichev, A.G., Sekii, T.: 2010, High-Resolution Helioseismic Imaging of Subsurface Structures and Flows of a Solar Active Region Observed by Hinode. Astrophys. J. 708, 304-313. doi $10.1088 / 0004-637 \mathrm{X} / 708 / 1 / 304$

Zhao, J., Couvidat, S., Bogart, R.S., Parchevsky, K.V., Birch, A.C., Duvall, T.L., Beck, J.G., Kosovichev, A.G., Scherrer, P.H.: 2011, Time-Distance Helioseismology Data-Analysis Pipeline for Helioseismic and Magnetic Imager Onboard Solar Dynamics Observatory (SDO/HMI) and Its Initial Results. Solar Phys., 163. doi:10.1007/s11207-011-9757-y

Zweibel, E.G., Gough, D.O.: 1995, Is There a Seismic Signature of the Sun's Magnetic Field? In: Helioseismology, ESA Special Publication 376, 73 
SOLA: sdo3_astro-ph.tex; 30 October 2018; 0:45; p. 42 